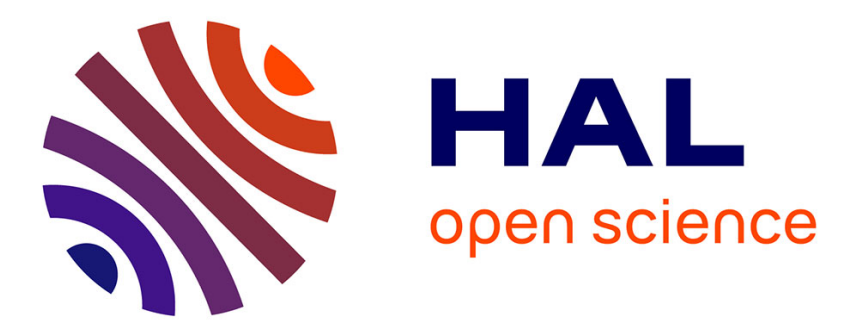

\title{
Les tombes protohistoriques de la bergerie Hermet à Calvisson (Gard)
}

\author{
Bernard Dedet, Michel Py
}

\section{To cite this version:}

Bernard Dedet, Michel Py. Les tombes protohistoriques de la bergerie Hermet à Calvisson (Gard). Gallia - Fouilles et monuments archéologiques en France métropolitaine, 1973, 31 (1), pp.37-59. 10.3406/galia.1973.2624 . hal-01926385

\section{HAL Id: hal-01926385 https://hal.science/hal-01926385}

Submitted on 4 Feb 2020

HAL is a multi-disciplinary open access archive for the deposit and dissemination of scientific research documents, whether they are published or not. The documents may come from teaching and research institutions in France or abroad, or from public or private research centers.
L'archive ouverte pluridisciplinaire HAL, est destinée au dépôt et à la diffusion de documents scientifiques de niveau recherche, publiés ou non, émanant des établissements d'enseignement et de recherche français ou étrangers, des laboratoires publics ou privés.

\section{(이) $\$$}

Distributed under a Creative Commons Attribution - NonCommercial - NoDerivatives| 4.0 


\title{
LES TOMBES PROTOHISTORIQUES DE LA BERGERIE HERMET A CALVISSON (Gard)
}

\author{
par Bernard DEDET et Michel PY
}

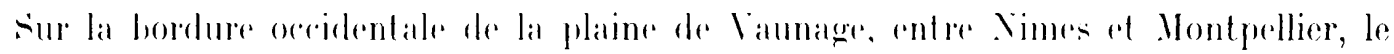
territoire de la commune de Calvison recele entere autres de nomberux vestiges proto-

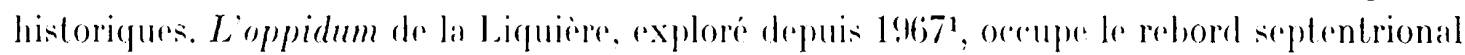
d'un grand plateau limitant a l'ouest la launage. Au sud de ce plateau, un serond oppidum a ele repére et a fait lobjet de premiers sondages en 1970 : il sagit de l'habitat de la Fonl

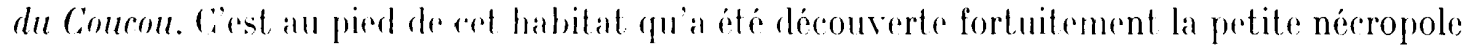
que concerne cette note (figr. 1 at : 2$)$.

La Font du Courou, a laquelle l hablitat doit son nom, sourd sur le llane sud du mamelon qui porte l'oppidum, vers une combe profonde qui se dirige vers le sud, et à la naissance de languelle se trome un petil hassin alu milieu de la garrigue. A partir de ce bassin, vers le courhant, samorere un deuxieme vallon fig. 1). Ine partie de res lieux avait eté récemment plantere en vigne (Calvison, parcelles carlastrales 151-197), et une autre devait

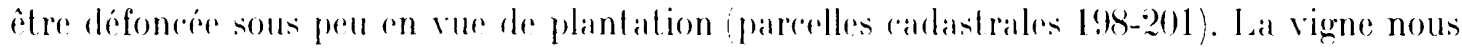
ayant livré de nomberux tessons lypiques du Dailhacien I, nous arons procéde a un sondage dans la partie encore intacte ef menacere. Le sondage, qui a consiste en une tranchée

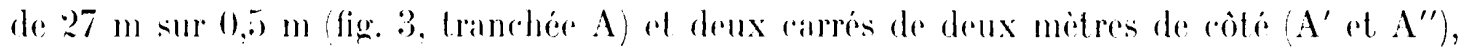
a rencontré principalement les restes non en place d'un habitat de type mailharcien I. Cies témoins seront étudiés ultérieurement.

En juillet 1970. le propriétaire a procéde au defoncage du terrain ou les sondages avaient ete cffectues. Les travaux ont bouleverse le sol jusqua une profondeur variant entre 0,60 el $0,80 \mathrm{~m}$. La prospection de surface, apres le defoncage, nous a permis de:

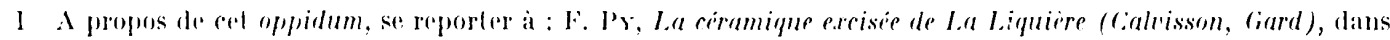
Cellicum XXIII, Congris de Tarhes 1969 'sous presse, F. P'Y, La eiramique corinthienne de la Liquiire (Callisson,

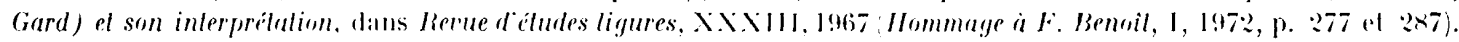

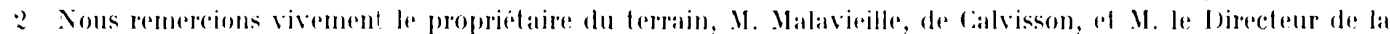
Circonscription des anliduites bistorigues du Languedoc-Roussillon qui nous onl fourni le's aulorisations necessaires,

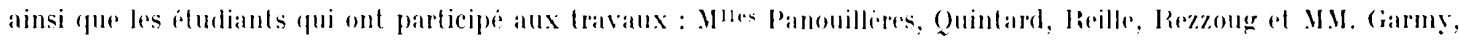
Niarf, F. Py et P. Py. Ce dernier a assure l'illustration photographicque de co Iravail. 


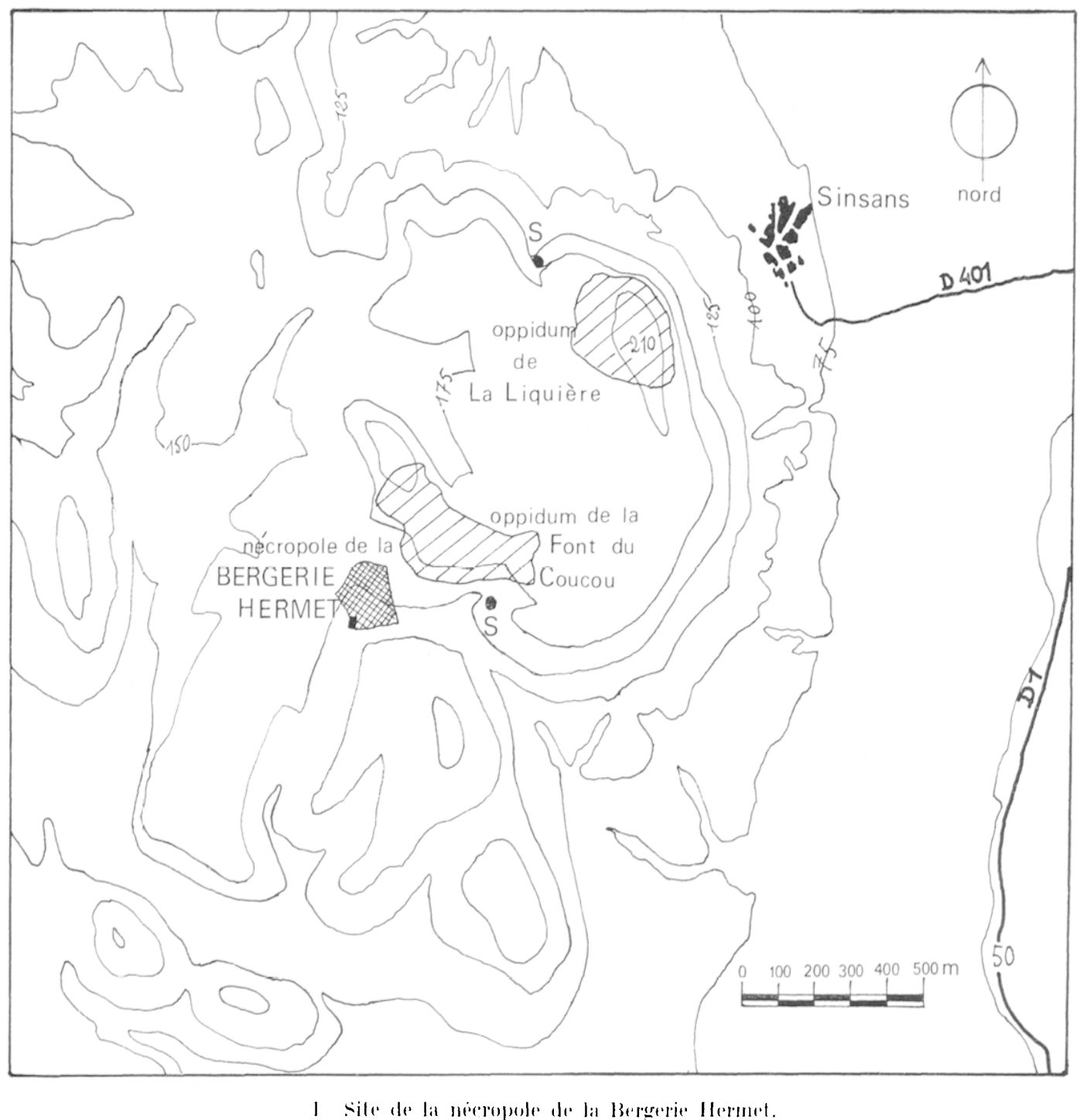

recurillir un tres abondant materiel de type mailhacien l. provenant de l'habilat repere précedemment. Nous notons on harbure sur la figure :3 la repartition de ces f rouvailles. Au nord, a l'est et a lourest. l’érosion avait emporti les sédiments antiques ef la charrue a soulere les strates du substrat rocheux. Au sud au contraire, il sest produit une aremomulation de terre contre le mur séparant la vigne de l'herme. et le soc na pats rencontré les rourhes arehéologiquess que lon redroure rependant plus bas dams lat vigme.

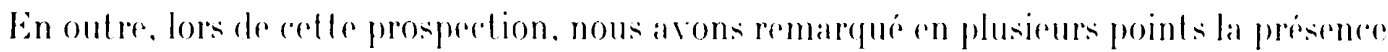
de fragments dos calcinés. groupes dans de petites mottes de cendres. Le tamisalge de la

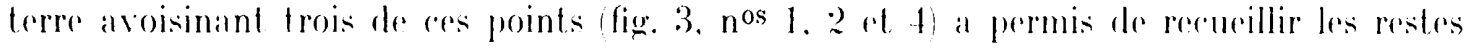
bouleversies de trois tombes. Sur un quatrieme point fig. 3, $\mathrm{n}^{0} 3$ 3) nous arons rencentré en profonde ur une tombe érété par la charrue dont les témoins átaient restés miraculeusement groupes. Le materiel des tombes est netlement plus récent que le gisement mailhacien I qui les contoure, at forme de ce fait un ensemble a part qu'il nous a paru utile dechulier séparément iri. 


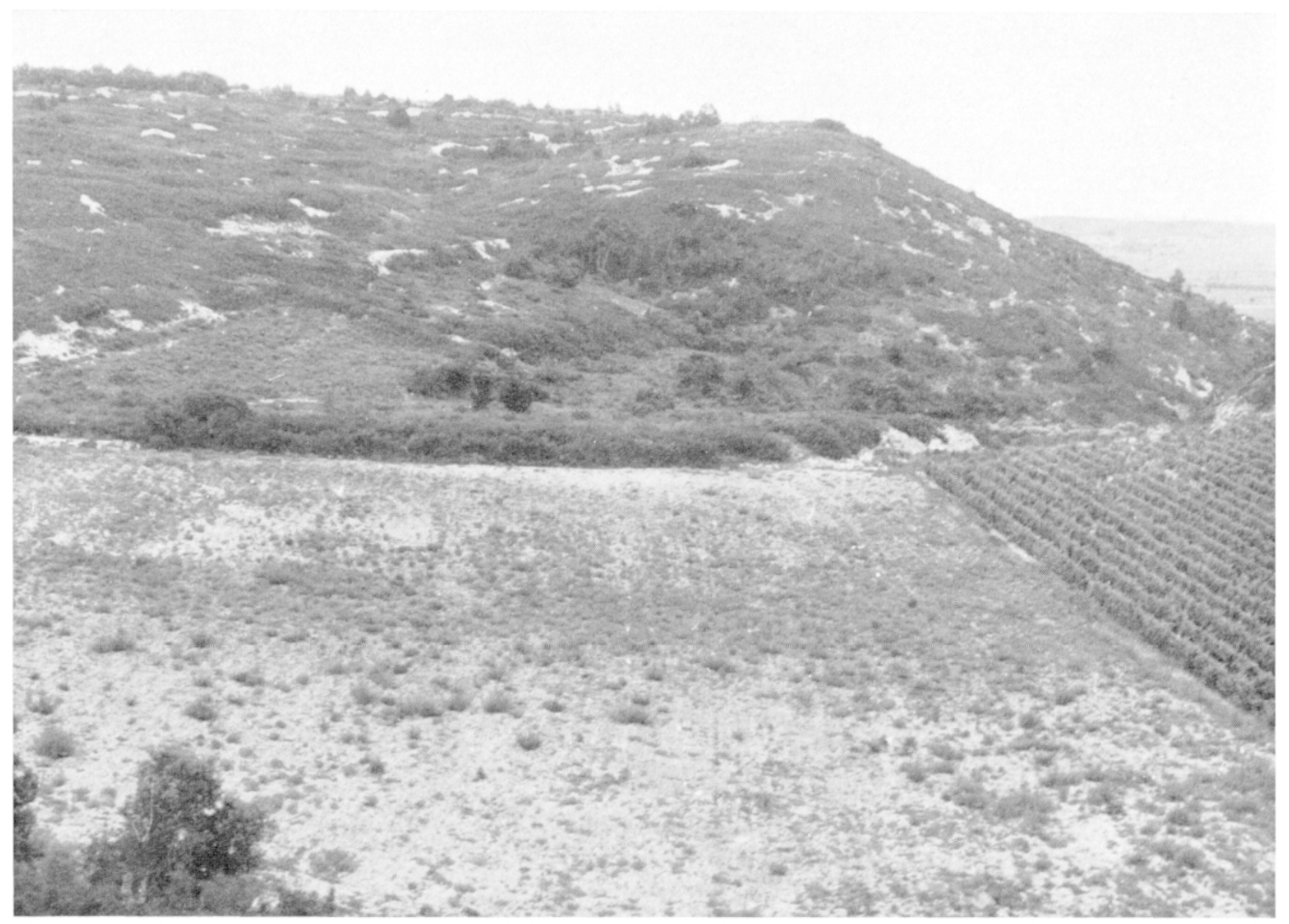

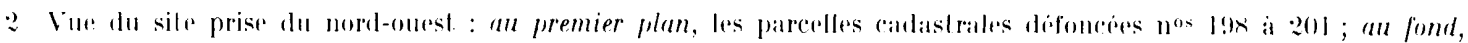
loppislum de lat font du roucont.

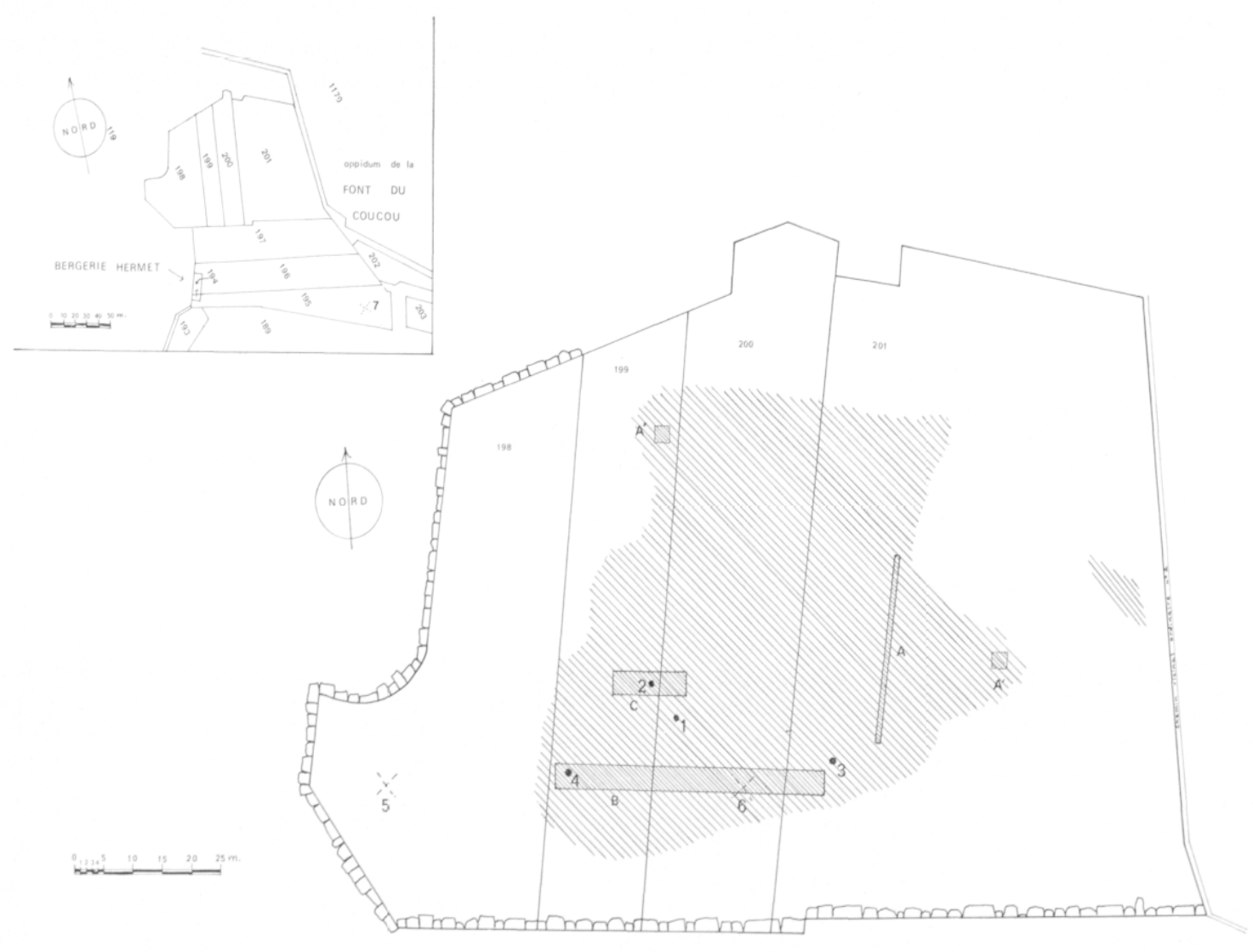

3 Extrait du plan cadast ral on siluation des tombes. 


\section{DFATRIPTON DES TOMBES}

Tombe 1. Elle était completement bouleversée. Ia charrue était descendue plus bas que le fond du loculus, si lien que le sondage a rencontré le rocher directement sous la couche de terre remuée. Les restes de la tombe se presentaient sous la forme de quatre petits tas de cendres et de charbons, mêlés d'ossements humains calcinés ${ }^{3}$, de débris de réramiques et de fragments métalliques.

MobiLier : nous avons recueilli les restes d'un vase non tourné, de deux vases tournés el d'un routeau en fer. Le tamisage ayant été effectué sur $9 \mathrm{~m}^{2}$, aux alentours de ce point de découverte, il semble que la quasi-totalité du matériel conservé dans la lombe avant le défongage ail élé récupérée.

$1^{0}$ Céramique non lournée ${ }^{4}$ : fragments de col et de panse d'urne décorée, à panse surhaussée et profil arrondi (inv. Cis/1) (fig. 4, no 3 ). Le dégraissint, tris abondant, est composé presque exclusivement de calcaire brové. Ces inclusions sont mal calibrées, fines et moyennes (jusqu'ia 0,002 m); elles sont mal réparties. lee col el la panse présentent le mème lissige fini (état de surfare $n^{\circ}$ bj $j^{5}$, lant a l'intérieur qu'a l'extérieur. Les surfaces extérieures. intérieures el la cassure présentent la mème couleur chamois, partout homogene. Ie deror est fail d'une rangée horizontale d'impressions profondes, obliques, étroites el obtenues par déplarement de l'outil (longueur de chaque impression $0,0(0) \mathrm{m})$.

20 Céramique allique : une coupe à rernis noir presque complete (inv.. Cos/4) (fig. 4, no 1 et fig. j). Il ne manque que les anses et la partie haute du pied. La pate est rosée et devient par endroit, jaune-orre ì brun sous l'eflet d'un feu secondaire; elle est line, dure lorsqu celle n'a pas été brûlée, et présente des cassures nettes. L'argile est rayable a l'ongle mais ne laisse pas de traces au toucher. Dans les parties brùlées, elle se fail tendre, friable, el présente des cassures émoussées. Le dégraisiant est constitué par une très line poussière de mica blanc. Le vernis se prisente de mème différemment selon l'élal de conservation des lessons. Les fragments les mieux conservés, qui n'ont pas subi l'épreuve du feu lors de l'incinération, ont un vernis de bonne qualité fait de lrois courhes successives: la premiire a une couleur rouge-orange et un aspect mat, la seconde couche, mate également, a une couleur brun-rouge; la troisieme courhe est noir profond. Elle est lisse, luisante et présente quelques irrisations brillantes. Ce vernis rouvre l'ensemble de lat roupe a l'exeption du plan de pose, de l'intérieur et du bourrelet externe du pied. Ies trois courhes de vernis sont particulierement fines et passées au pinceau aver soin. Il s'agil d'une coupe allique a levere incurvée du lype $C^{6}$.

30 Cierramique grecque doccident : un vase support a pied tres haut et vasque tris basse et

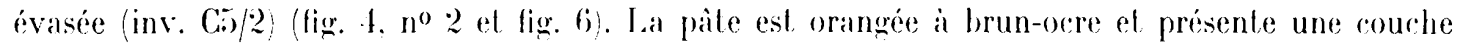

3 Pour l'alude des mestes humains, voir lannexe ridige par H. Muday, que mous remereions ici.

4 Vous employons le terme de criamique non lourne plulot que l'une des trois appellations les plus courantes

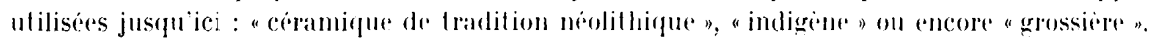

5 Cous distinguons cind phases dans laménagement de ba surface des vases non tournes : élal de surface $n^{\circ} 1$ :

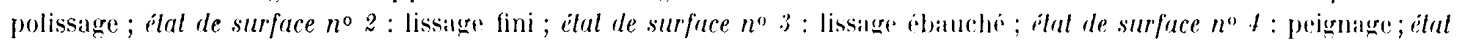

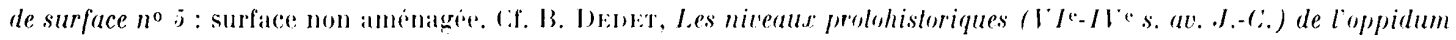
de Vié-Cioutat (.Mons-.Vonteils, Ciard), Campagnes de foulles 1966j-1968, dans Revue archélogique de .Varbonnaise isous presse'.

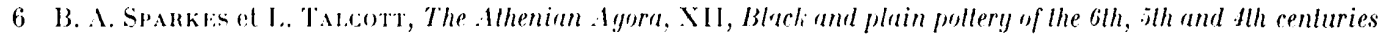
B.C., 1970, 1, p. 91 . 

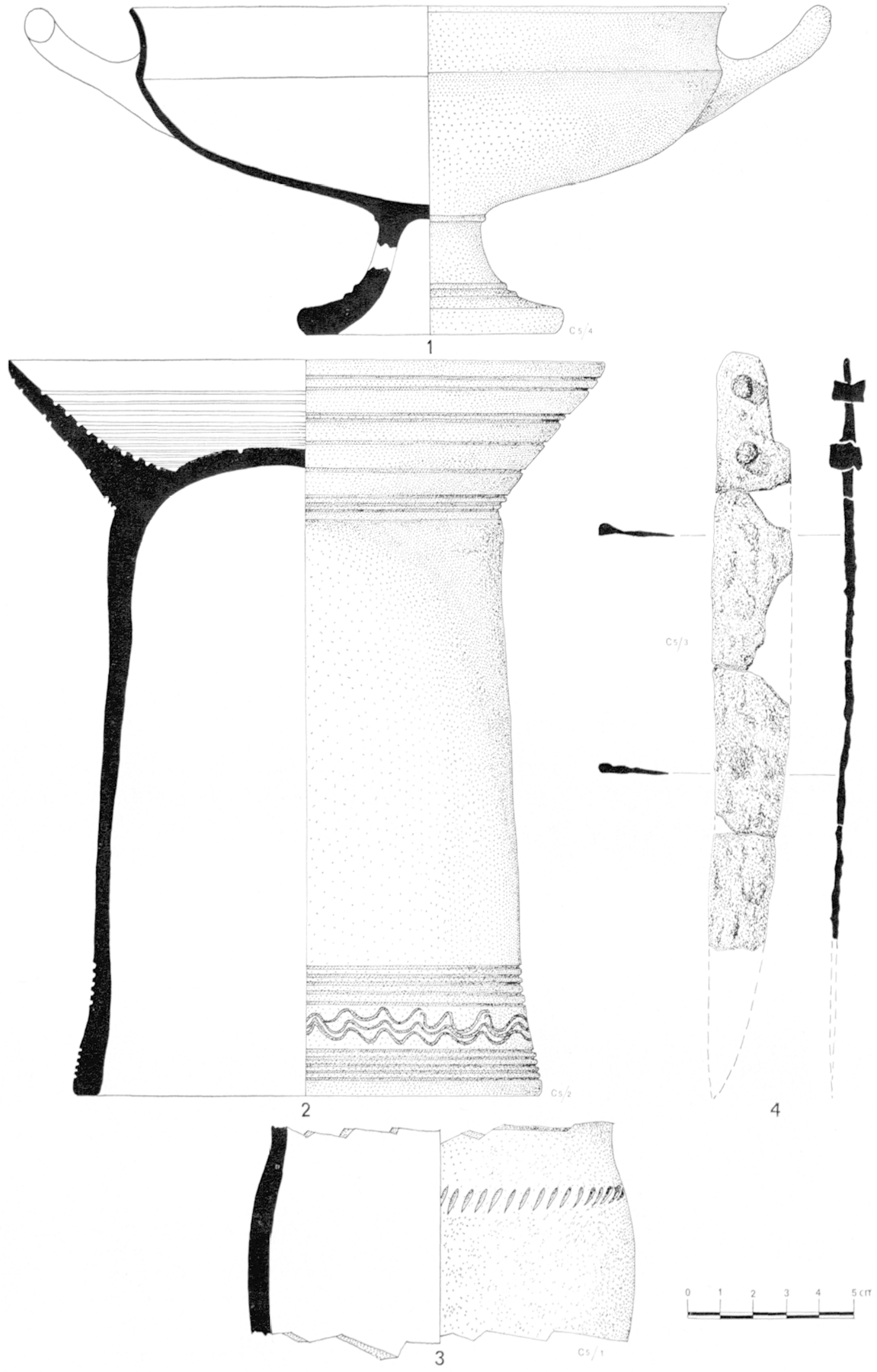

4 Mobilier de la tombe $1: \mathrm{n}^{\circ} 1$ : coupe altique; $\mathrm{n}^{\circ} 2$ : vase support con ceramique grecepue docrident ; $1^{\circ} 3$ : fragments d'urne non tournee; $n^{\circ}+1$ : coutrall in fer. 


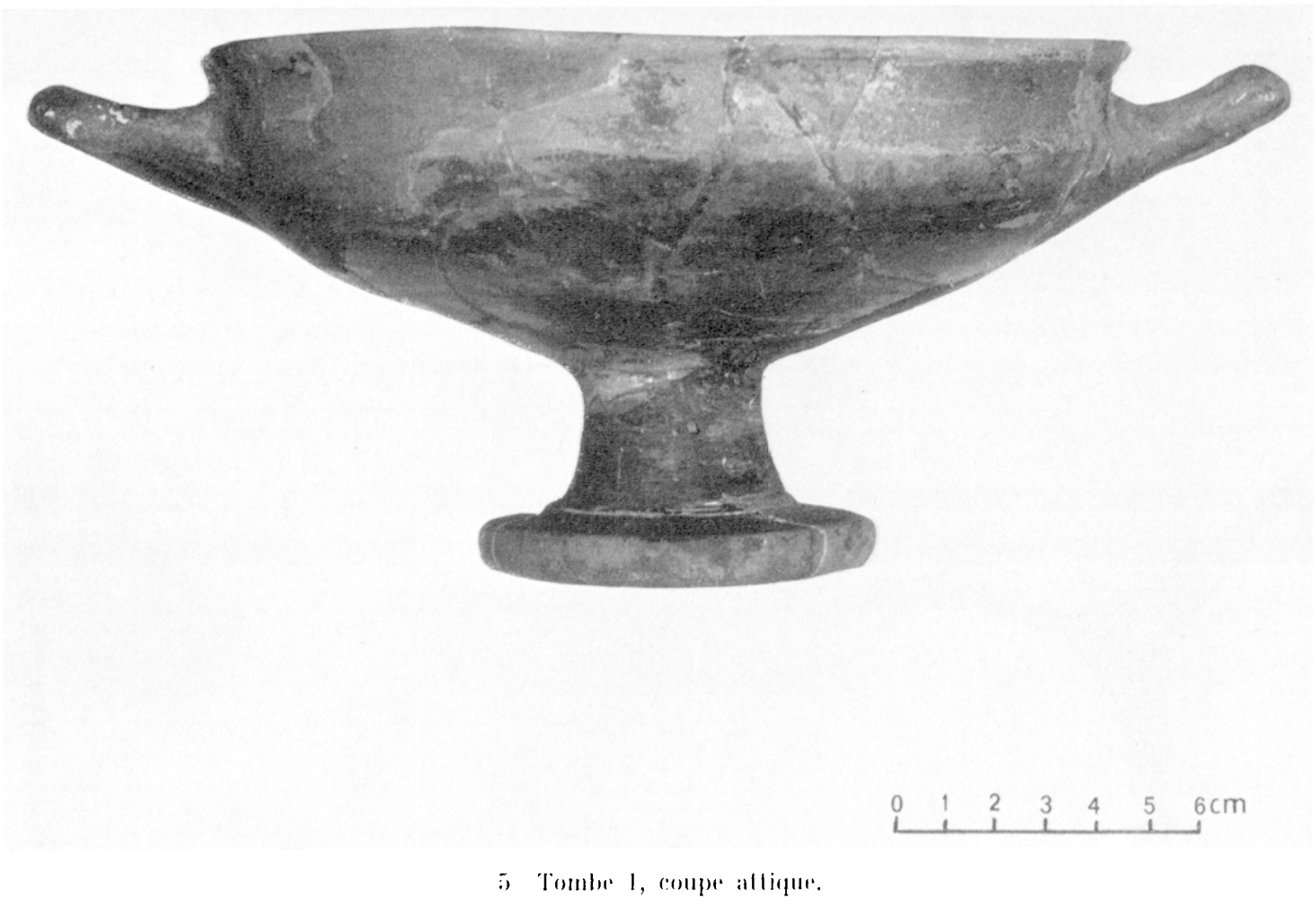

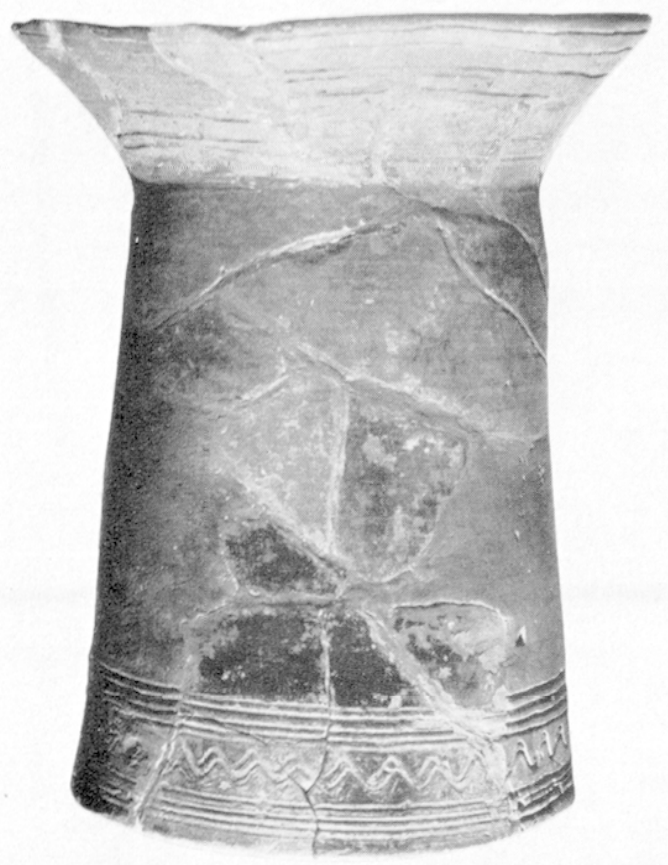

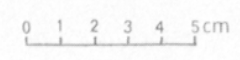

6 Tombe 1 : vase support en ceramique erecepue 1) (occident. d'argile interne de couleur ocre. La lexture en est homogine mais tres varuolaire. L'argile est assez fine, un peu rugueuse, fendre el rayable à l'ongle. Elle laisse des traces au lourher. Ie degraissant est compose de mica fin, blane el de quelques parcelles calcaires. La peinture est en partie effacée. Elle rourrait a l'origine la face externe du pied principalement. Sa couleur est jaune-ocre. Elle devient brun-noir dans les parlies brûlées secondairement. C'est une couverte épaisse, composie? a base d'argile. Ia vasque porte, aussi bien a l'exterieur qu'a l'interieur. un décor de slries paralleles et horizontales. Celles-ci sont régulierement réparties a lontérieur. el inégalement. groupées a l'exlérieur. A la base du pied, prend place une onde irréguliere, faile gràce à un peigne a trois dents. comprise entre deux groupes de cing stries parallides et horizontales. Ce vase, si ce n'est par la couleur de la pale, entre dans la categorie grise monochrome locale.

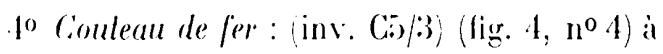
soie portant deux rivets et dont il manque la pointe. La lame présente un seul tranchant. Ia forme générale est légrement arquée. 


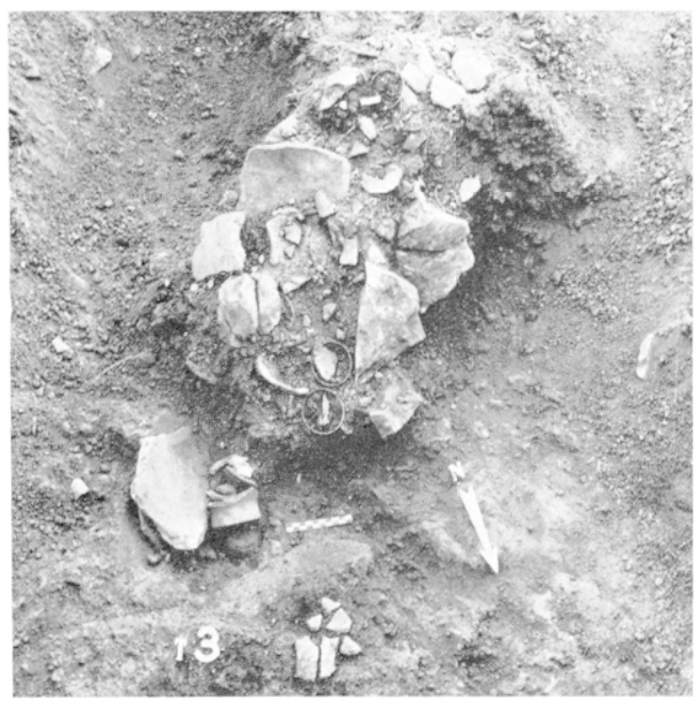

7 Tombe 3, rate de la fombe apries le passage de la charrur.

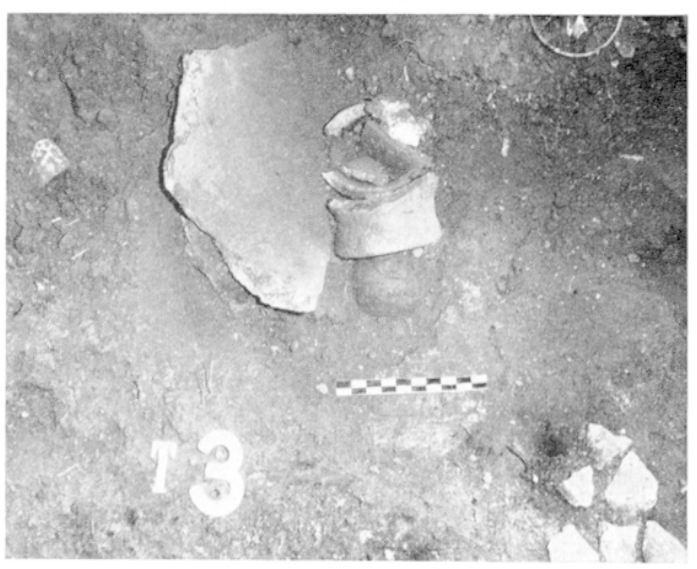

$x$ Tombe 3, detail du fond du loculus.

Trombe 3. Elle était la moins boulerersé de toutes fig. 7). Située entre deux sillons, elle avait été en partie renversée par la charrue. Le soc était passé a la limite nord de la tombe, at les vases avaient éclaté et araient été déportés vers le sud. Mais l'ensemble du matériel etait resté groupé. La charrue neetait pas deseendue plus bas que le loculus, de sorte que le fond de ae dernier a pu ètre localisé. Il portait encore l'empreinte de l'emplacement de deux vases (fig. 8) et se situait à $0,80 \mathrm{~m}$ environ sous le sol actuel. Il semblait brùlé et durei. Tout autour du mobilier et dispersées sur $4 \mathrm{~m}^{2}$, se trouvaient de très nombreuses pierres lorùlées.

\section{Momilis:r :}

10 Céramique non tournes : a) urne complete, à panse surhaussée et profil arrondi ; pied divergent bas ; col divergent haut a contact col-pante adouci ; bord incliné vers l'extérieur à lève arrondie (inv. C2:/32) (fig. 9. $n^{\circ}$ 5) el fig. 10). La hauleur est de 0.17\%) m et le rapport de la hauteur sur le diamètre miximum du vase es 1:31. Le dégraissint. composé en proportions sensiblement égales de calcaire brové. de dolomie et de quart\%, est très abondant, fin (jusqu'a 0,001:3 m), bien calibré, mais mal réparti. Les cassures sont irrégulieres et la forme semble avoir été obtenue par reusement et étirement de la molte d'argile. Le lissage est fini (élat de surface $n^{0} 2$ ) aussi bien sur le col que sur la panse. la surface extérieure varie de l'ocre-rouge au brun-noir. La surface intérieure est brun-gris homogène. La cassure montre une large zone centrale d'un noir homogine encadrée de deux bandes orres vers les surfaces. Ce vase ne porte aurun décor.

b) Lrne complete a panse surhaussée et profil arrondi f fond plat; ; col divergent haut a contact col-panse adouci; bord incline vers l'exlérieur a levre arrondie (inv. C2:/3:3) (fig. 9, no 6 et fig. 11). La hauteur est de 0,13:3 m et le rapport de la hauteur sur le diamitre maximum du vase est 122. Le dégraissint. composé presque exclusivement de calcaire et de calcite broyés, est très abondant, mal calibré (jusqua i 0,00:3) mi et mal réparti. Les cassures irrégulieres suggerent un vase creusé a partir de la motle. Les surfares exlérieures et intérieures, bien qu'actuellement presque partout altérées, laissent rependant voir un lissige fini (etat de surface $n^{0} 2$ ), aussi bien sur le col que sur 


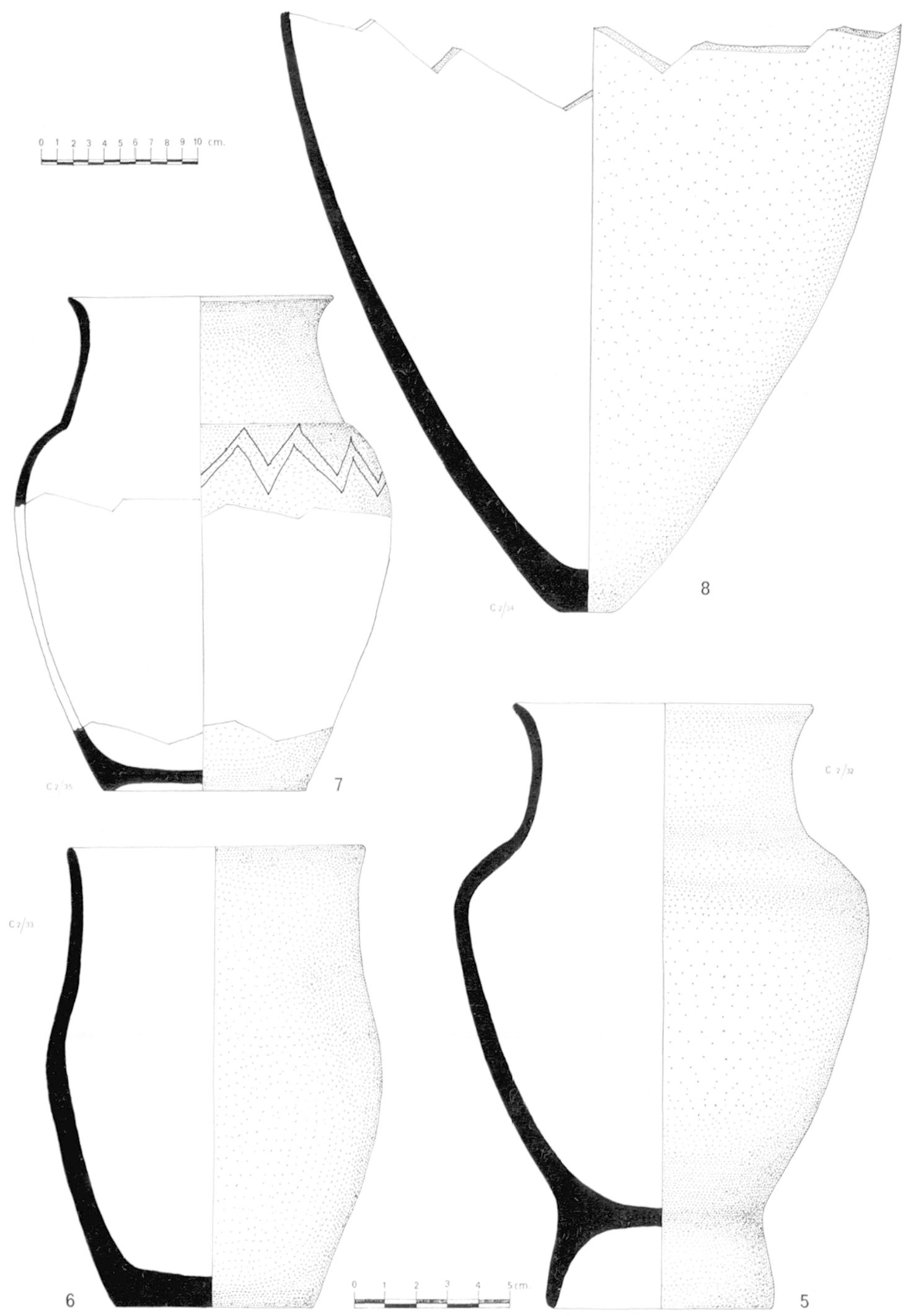

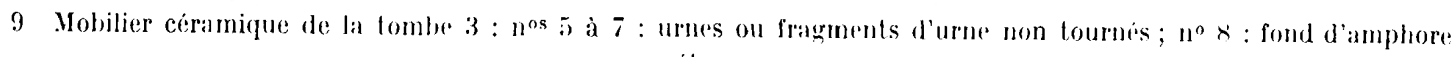
it rusque. 


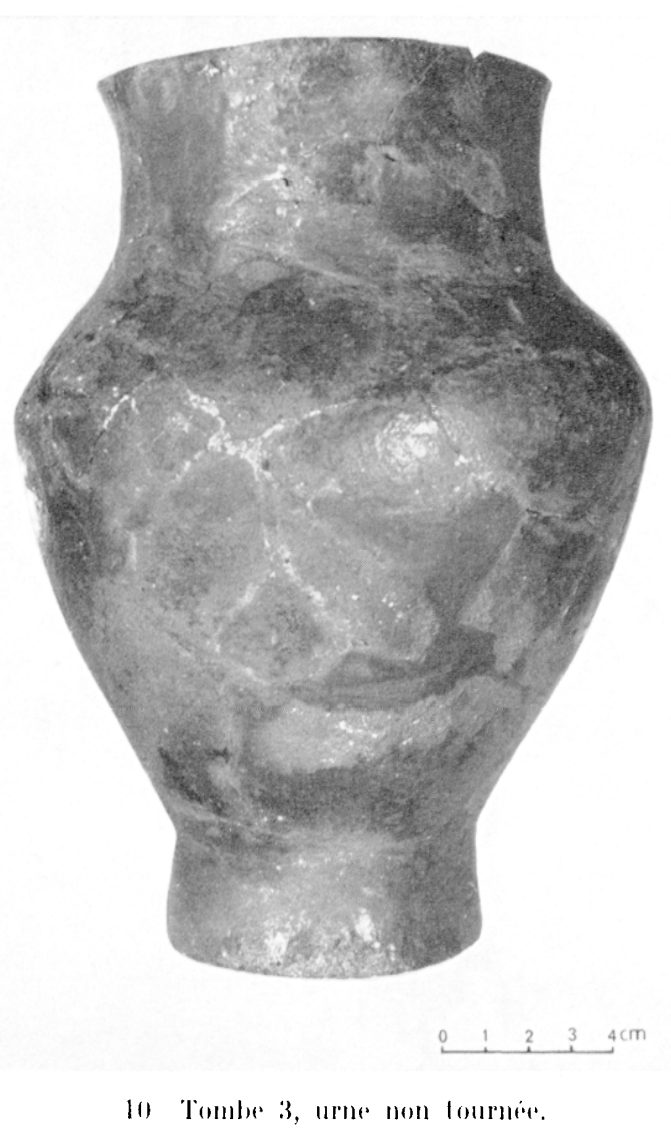

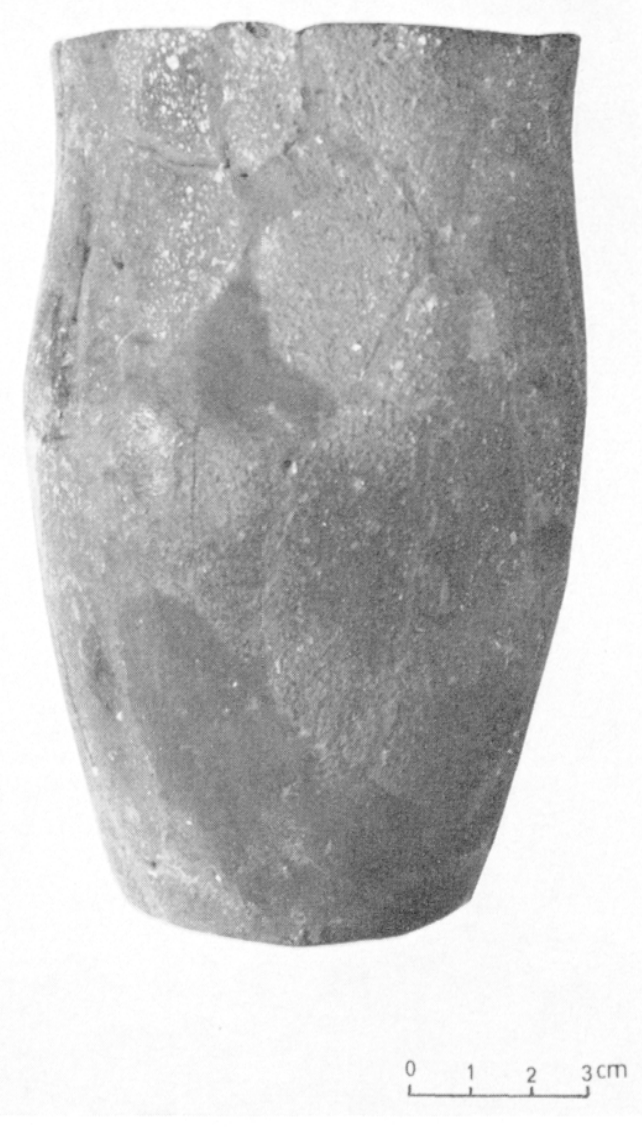

11 Tombe 3, urne non tourneve.

la panse. A l'extérieur la couleur de la surface varie du chamois au gris-noir, tandis que l'intérieur est d'un gris homogine. L'épaisseur est de couleur gris-noir au centre, ocre sur les zones superfirielles. Ce vase n'est pas décoré.

c) Crne fragmentaire a panse surhatusie el profil arrondi ; fond reux ; col converont hatut, a contact col-panse anguleux ; bord incliné vers l'extérieur a levre arondie frol, épaule, fond, seuls conservés) (inv. C2/35) (fig. 9, no 7). L'état de conservation ne permet pas de calculer la hatuteur ni le rapport de la hauleur sur le diametre maximum. Ce rapport devait rependant se situer aux alentours de 100. Le dégraissant est uniquement romposé de calcaire broyé Iress fin (jusqu'a $0,0012 \mathrm{~m}$ ). Il est assez abondant, bien calibré et semble bien réparti. Le lissage est fini (élal de surface $\left.\mathrm{n}^{0} 2\right)$ aussi bien sur la surfare du col que sur celle de la panse a l'extérieur qu’a l'intérieur. I a couleur des surfares extérieures et intérieures passe de l'ocre-beige au brun-gris. La cassure présente une large zone centrale noir homogene, encadrée de deux zones superficielles orre-gris. I de décor. situé sur l'épaule, se compose de deux lignes brisées superposées, faisant le lour du vase. Ces deux lignes, tracées séparément, sont incisées a la pointe mousse.

2o Céramique lommé : fond d'amphore étrusque, pointu et présentant un trés léger aphatissement de la pointe inv. $\mathrm{C} 2 / 3.3$ t tig. 9. no 8 . Les parois, relativement minces. montrent interieurement des traces de tour. la pale est dure, de couleur brun-rouge sur les fares exlernes de la paroi, el brun-noir a noir a l’interieur de celle-ci. Le dégraissant. abondant. est a hase de sable. Il contient des grains de quart\% el surtout une importante proportion de paillelles et de grains de mica noir. Ce degraissant rend la pàte rugueuse au toucher. 

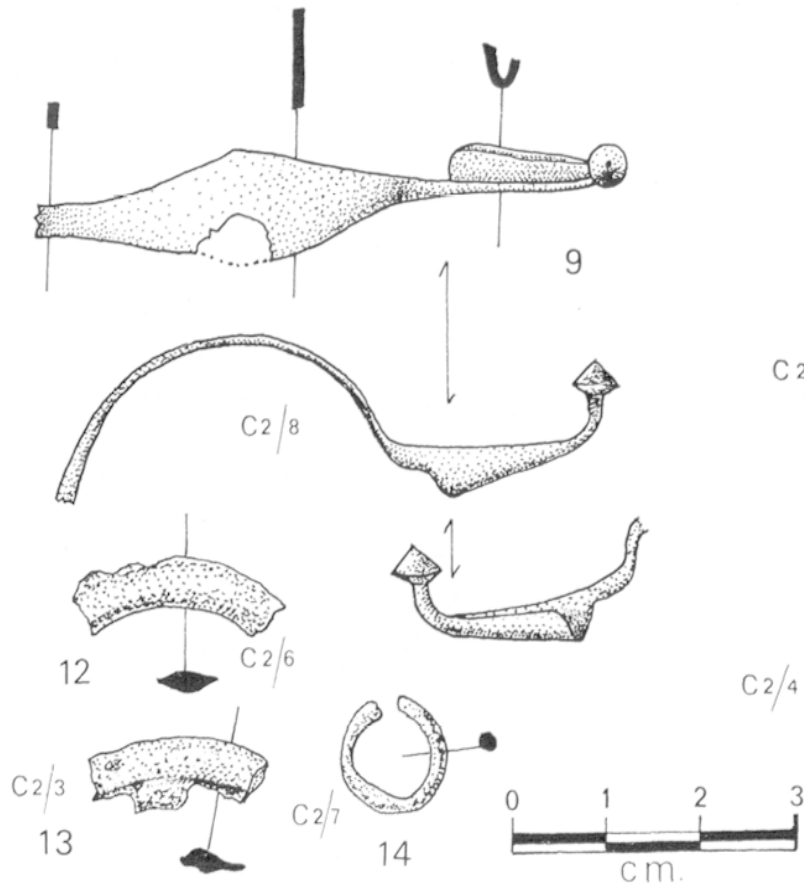

12

Mobilier medallique de la tombe $3: n^{\circ} 9$ : fibule en bronze; nos 10 à 1.1 : ammeaux el fragments dameaux en liromze.

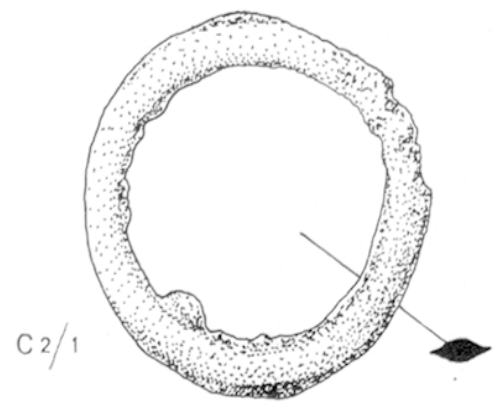

10

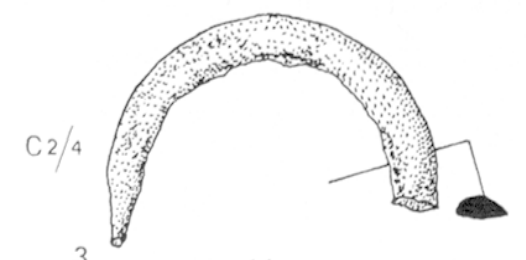

11

13 Tombe 3, fibule en loron\%e fichelle 1:1.

$3^{0}$ Mobilier mélallique: a) quatre fragments d'une mème fibule de bronze (inv. C2/8) (figg. 12), $n^{0} 9$ et fig. 13) dont il manque le ressort et l'ardillon. L'arc est à profil arrondi ; sa section est aplatie. Le porte-ardillon est long ; le pied est relevé et bas; il se termine par un boulon conique dont la base a une arète vive (Iongueur totale conservée : $0,062 \mathrm{~m}$ ).

b) Huit bracelets de bronze complets (fig. 14 et 15) et de tris nombreux fragments correspondant à un minimum de 14 autres bracelets du même métal. Tous les exemplaires complets sont fermés. Ils sont tous du type armille, fait d'un trés mince ruban plat. Ie décor, lorsqu'il existe, est toujours très usé, parfois même en grande partie effacé et n'affecte que la face extérieure.

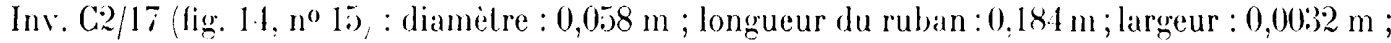
épaisseur : $0,001 \mathrm{~m}$; section reclangulaire ; exemplaire non décoré.

Inv. C2/18 (fig. 14, no 16) : diamètre : 0,06;3 m ; longueur : 0,20 m ; largeur : 0,00:3 m ; épaisseur : $0,001 \mathrm{~m}$; section rectangulaire. I.e décor semble consister en groupes de sept incisions lines; ces groupes sont obliques et d'orientation opposée.

Inv. C2/19 (lig. 14, no 17) : diametre : $0,06.3 \mathrm{~m} ;$ longueur : $0.20 \mathrm{~m} ;$ largeur : $0,0028 \mathrm{~m}$ sur la face externe, et $0,00: 31 \mathrm{~m}$ sur la face interne; épaisseur : $0,0012 \mathrm{~m}$; section trapézoïdale; le décor tres effacé est semblable à l'exemplaire précédent.

Inv. C2/20) (fig. 14, $\mathrm{n}^{0} 18$; : diamètre : $0.063 \mathrm{~m}$; longueur : $0,198 \mathrm{~m}$; largeur : 0,0025 m à 


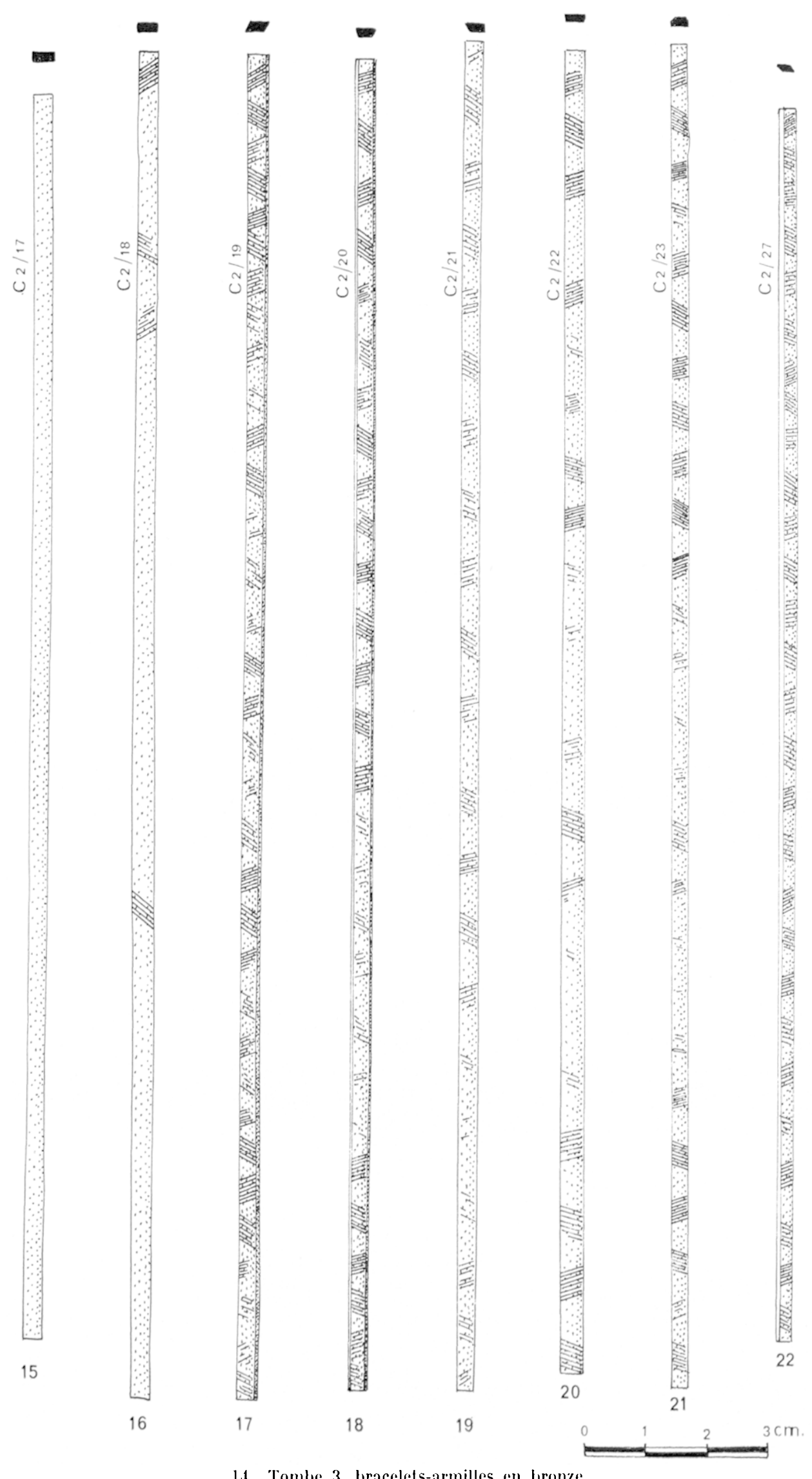

1.1 Tombe 3, bracelets-armilles en bronze. 

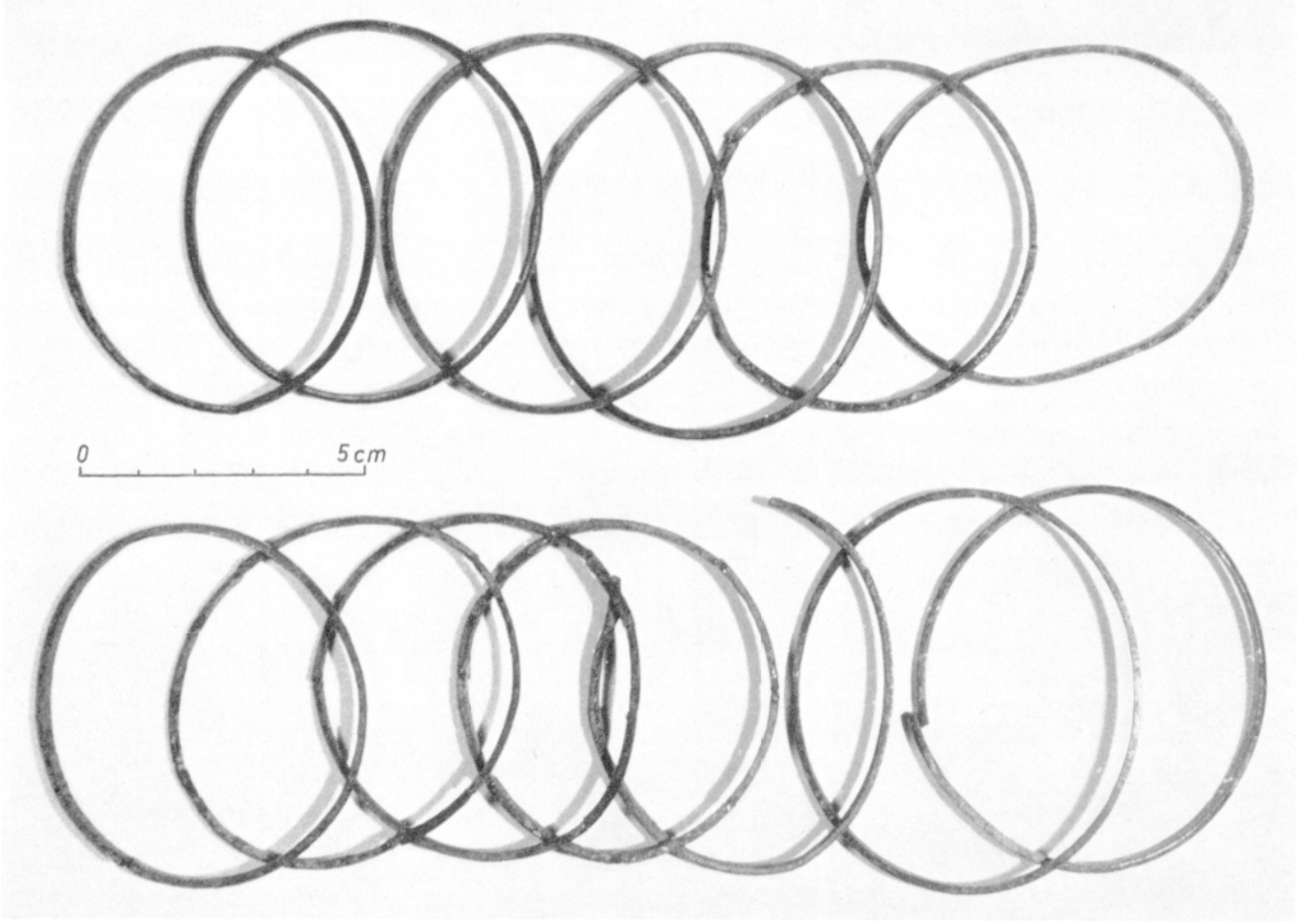

15 Tombe 3, bracelets-almilles an bronze.

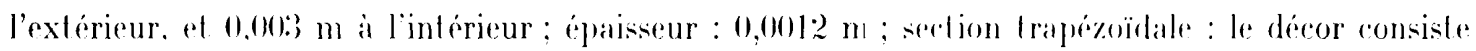
en groupes obligues de huif incisions fines d'orientalion opposien.

Inv. C2/21 (lig. 14. $\mathrm{n}^{0} 19$ : diamitre : $0,0633 \mathrm{~m}$ : longueur : 0,20$) \mathrm{m}$; largeur : $0,0003 \mathrm{~m}$ vers l'extérieur, el (1,012:) m vers l'intérieur ; épaisseur : $0,0012 \mathrm{~m}$; section trapézoïlale; il ne reste que quelques traces du décor. formé de groupes dincisions fines, groupes d'orientation opposée.

Inv. C2/22) (fig. 14. no 20 : diamitre : $0,0633 \mathrm{~m}$; longueur : $0,196 \mathrm{~m}$; largeur : $0,00135 \mathrm{~m}$; épaisseur : 0,0012 m; sertion rectangulaire; le décor est formé de groupes obliques et d'orientation opposée de 6 a 7 incisions lines.

Inx. C2/23) (fig. 1.4. $\mathrm{n}^{0} 21$ : diamele : $0.066 .3 \mathrm{~m}$; longueur : $0,20 \mathrm{~m}$; largeur : $0,0028 \mathrm{~m}$ à

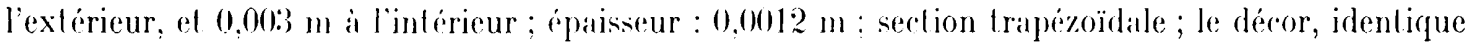
à celui des exemplaires décorés précédents, comporte des groupes de 6 a 8 incisions.

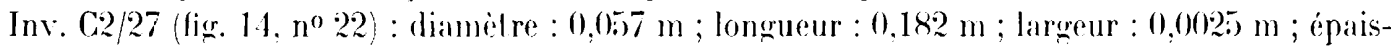
seur : $0.0012 \mathrm{~m}$; section losingrique; le décor est identique.

les nombreux fragments de bracelets-armilles ont une largeur qui varie de $0.00 \%$ a a a $0.0015 \mathrm{~m}$;

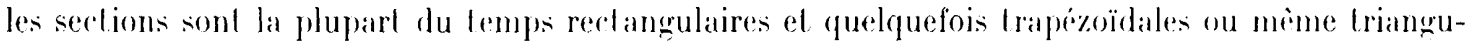
laires.

c) Qualre fragments de brarelet double a surface lisse, en bronze.

d) In anneau lisse en lironze. a sertion en fuseau (figr. 12, $n^{\circ} 10$ ) d'un diamitre de 0,00037 $\mathrm{m}$; trois fragments du mème lype correspondant au minimum a deux aulres exemplaires (fig. 12, nos 11 i 13$)$. $\left.n^{0} 14\right)$.

e) I'n petil anneau ouvert. en bronze, de section ronde, et d'un diamitre de $0.012 \mathrm{~m}$ (fig. 12, 
Disposition des objets : nous connaissons la place originelle du fond d'amphore étrusque el du vase cinéraire dont nous avons retrouvé l'empreinte sur le fond de la tombe (figr. 8). Par rapport à ces deux points sûrs, la majorile du matériel se trouve déjetée vers le sud et vers le haut, notamment les fragments supérieurs de ces deux vases et la totalité des bracelets. Le contact des bracelets, des principaux groupes d'ossements calcinés el des tessons du vase $n^{\circ} \mathrm{C} 2 / 3 \mathrm{3} 2$ laisse penser que bracelets et ossements chaient contenus dans ce vase. La fibule a été trouvée au fond du loculus, au sud de l'ossuaire. La majorite des tessons du vase C2/33) se trouvait dans la partie nord de la tombe. A l'ensemble de ce mobilier étaient mèlées des pierres portant traces de rubéfaction.

Tombe 4. Elle etait completement boulerersece. Elle etait marquere par trois mottes de cendres groupies sur un metre carré environ. Quelques fragments de charbons de bois et d'ossements étaient mélés aux cendres, ainsi que le matériel.

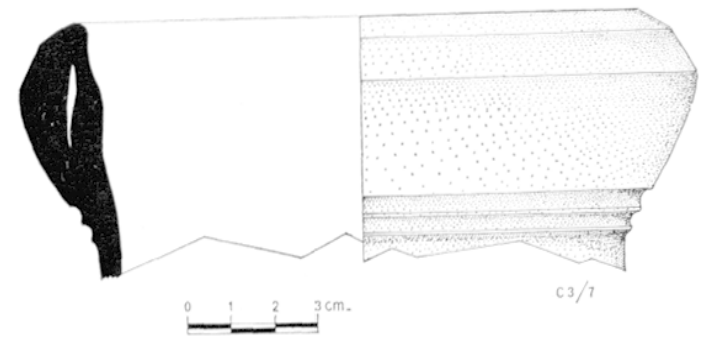

$\leftarrow 16$ Tombe 4, bord d'amphore de type ionien.

Mobihiner :

$1^{\circ}$ Céramique : un fragment de bord d'amphore sablée de type ionien (inv. C.3/7) (fig. 16), caractérisé par une lèvre repliće et soulignée de deux cannelures. Ia face extérieure de la lèvre présente deux facettes. La pâte, jaune-orre, est tendre ; le dégraissant, sableux, contient de nombreux grains de mica.

$2^{0}$ Mobilier mélallique : six bracelets-armilles complets, en bronze, tous fermés, plats, de section rectangulaire (fig. 17, 18 et 19. Cing bracelets sur les six portent un décor incisé sur la face externe. Sur tous les exemplaires, a la différence de la tombe 3, le décor est tris apparent. Il s'agit donc d'un lot moins usé que le précédent.

Inv. C:3/1 (fig. 17, $\mathrm{n}^{0} 23$ ) : diametre : $0,06 \mathrm{~m}$; longueur : $0.190 \mathrm{~m}$; largeur : $0,004 \mathrm{~m}$; épaisseur : $0,001 \mathrm{~m}$; cet exemplaire est décoré de groupes obliques et d'orientation opposée de 6 a i 7 incisions fines, de la même maniere que les bracelets de la tombe 3.

Inv. C:3/2 (fig. 17, $\left.\mathrm{n}^{0} 24\right)$ : diamitre : 0,06:3 $\mathrm{m}$; longueur : $0,198 \mathrm{~m}$; largeur : $0,004 \mathrm{~m}$; épaisseur : $0,001 \mathrm{~m}$; décor identique au précédent.

Inv. C:3/3 (fiq. 17, $\left.\mathrm{n}^{0} 25\right)$ : diametre : $0,062 \mathrm{~m}$; longueur : $0,195 \mathrm{~m}$; largeur : $0,003 \mathrm{~m}$; épaisseur : $0,000 \mathrm{~m} \mathrm{~m}$; ret exemplaire n'es pas décoré.

Inv. C:3/4 (fig. 17, no 26 : diamètre : 0,062 m ; lonqueur : 0,195 m ; largeur : 0,005 m ; epaisseur : $0,001 \mathrm{~m}$; le déror consiste en fines incisions obliques dans un sens, très rapprochées et couvrant toute la surface extérieure du brarelet, et en incisions plus larges, obliques mais d'orientation opposée et séparées par des intervalles de 0,002: a $0,003 \mathrm{~m}$.

Inv. C:3/5) (fig. 17, $\mathrm{n}^{0} 27$ ) : diamitre : $0,062 \mathrm{~m}$; longueur : $0,196 \mathrm{~m}$; largeur : $0,004 \mathrm{~m}$; ćpaisseur : $0.001 \mathrm{~m}$; le décor est identique a celui des exemplaires C.3/1 et C:3/2.

Inv. C:3/6 (fig. 17, $1^{0} 28$ : diamilre : 0,063 m ; longueur : 0,197 m ; largeur : 0,00 $1 \mathrm{~m}$; épaisseur : $0,001 \mathrm{~m}$; le décor est le mème que sur les exemplaires C:3/1, C.3/2 et C:3/5, rependant le nombre d’incisions par groupe est plus irrégulier et varie de a) a 7 . 
BERNARI IEDET ET IICHEA PY

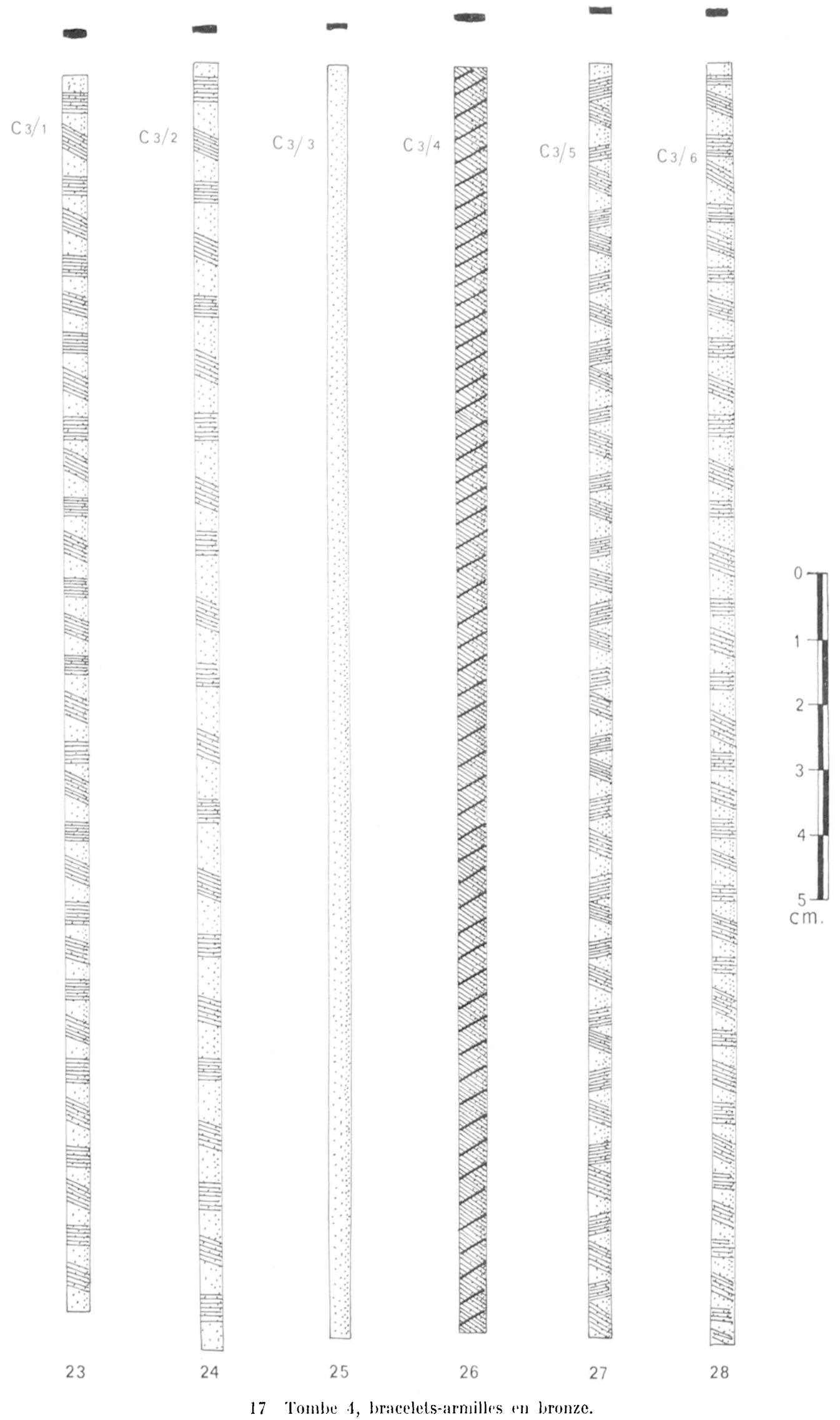




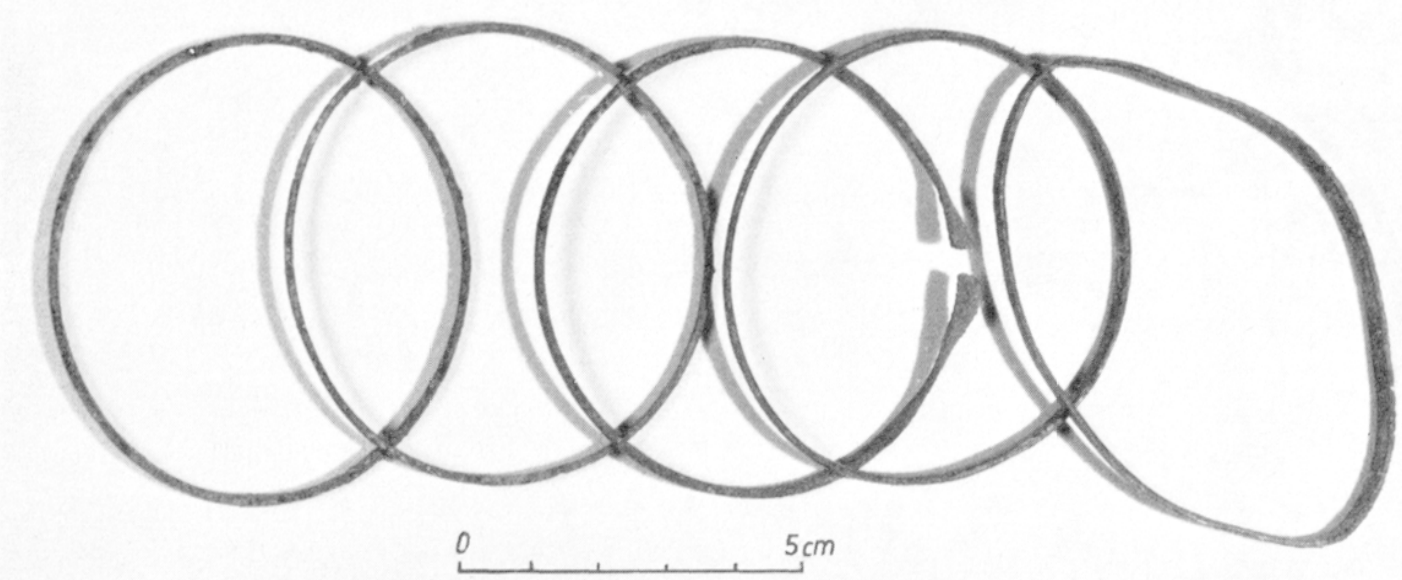

is lombe 4, bracelets-armilles en bronze.
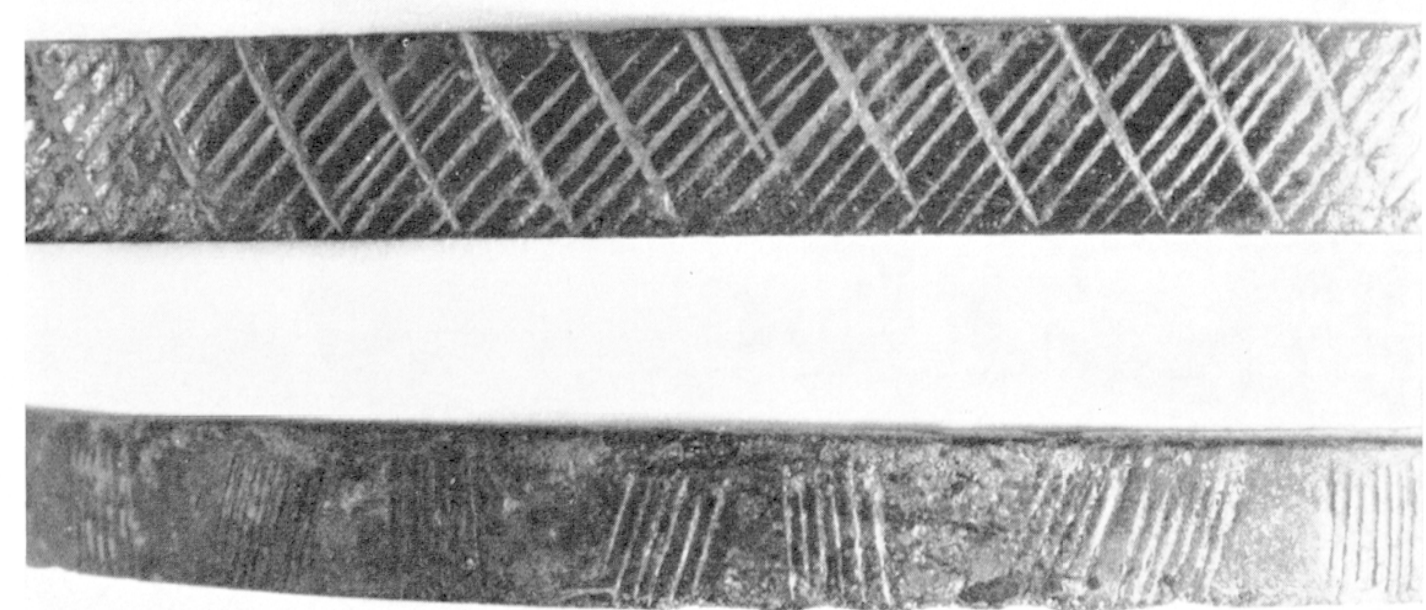

19 Tombe 4, delail du deeor des bracelels-armilles r: $3 / 4$ et cas/5.

Tombe 2. La ancore la tombe etait entièrement bouleversée. Dans deux petites mottes de cendress se trouvaient de nombreuses esquilles d'os calcinés at les débris éparpillés de deux rases.

\section{Mobilier :}

10 Céramique non tournée : urne complète a panse surhaussée et profil arrondi; fond plat; col divergent bas à contart col-panse anguleux; bord incliné vers l'extérieur à un méplat interne; lère arrondie (inv. CA/1) (fig. 20, no 29) ; la hauteur est de $0.14 \mathrm{~m}$. et le rapport de la hauleur sur le diamètre maximum est 89. Le dégraissant, composé pour moitié de calcaire et pour moitié de

7 Les quelques fragments dossements humains provenant de cetle tombe ne permettent pas d'analyse. 


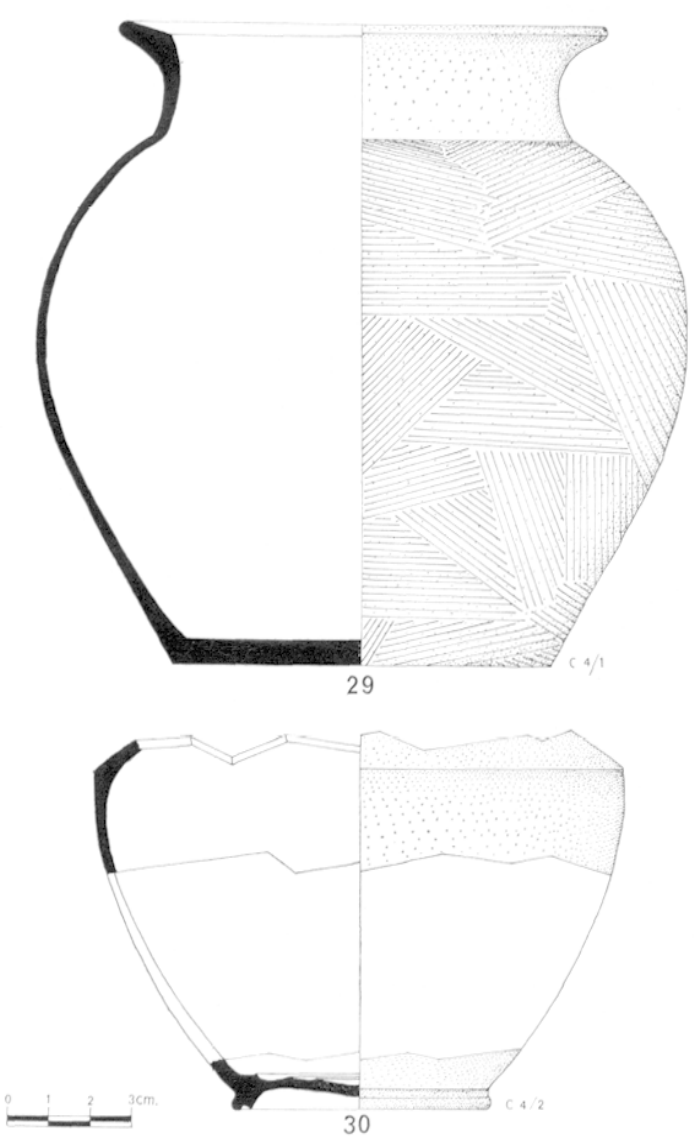

20) Mobilier cramicaue de la fombe : $:$ no $29:$ urme non toumere; no 30 : fragments dobpe en ceramique commune à patr claire. quartz et schisle, est asse\% peu abondant. Les inclusions sont fines et moyennes (jusqu'à $0,0025 \mathrm{~m}$ ), mal calibrés et bien réparties. La panse est irrégulierement peignée (elat de surface no 4) alors que le col montre un lissige fini (élat de surface $n^{0} 2$ ), ainsi (que l'intérieur (état de surface $\left.n^{0} 2\right)$. Ia couleur de la surface extérieure varie de l'ocre au gris, alors que la surface intérieure est uniformément ocre. L'épaisseur montre une couleur noire homogène. Ce vase ne porte pas de décor.

2o Cieramique loumée deux fragments, épaule et fond, inv. C4/2 (lig. 20, no 30) d'une olpé en ceramique commune a pâte claire. Ce vase présentait une épaule a carine bien marquée et un fond annulaire a rainure sous le plan de pose. Ia pate est jaune paille, rendue brun-yris par endroit sous l'eflet d'un feu secondaire. Elle est fine, homogene, lendre, laisse des traces au toucher el se raye facilement i l'ongle. On y remarque de rares inclusions de calcaire, a l'exclusion de tout mica.

Trourailles hors lombe. Ces trourailles ont cté faites en surface, parmi les très nombreux lessons datant do la periode Mailhareien I. Leeur date permet de les isoler.

10 Bord de coupe allique, inv. G1/209 (fig. 21, $\left.n^{0} .31\right)$ : la forme est semblable à la coupe de même origine trouvée dans la lombe 1. La pâte est rosée, asse\% dure, mais rependant rayable a l'ongle. Elle présente un dégraissant de mica blanc fin et abondant. Le vernis est formé de deux couches : la premire est lisse, de couleur rouge-brun; la seconde est luisanle. franchement noire et s'est conservée surtout a l'intérieur du vase. Cette couverte est de bonne qualité. La terhnique, la pate et le vernis sont tres prorhes de l'exemplaire de la tombe 1 .

20 Bord de compe ionio-altique. inv. C1/210 (fig. 21. no 32) : la forme est la même que dans l'exemplaire précédent, avec une levre plus retournce. La pâte est jaune-ocre, homogène, dure; les cassures sont nettes et la surface bien finie ; le dégraissant est formé de poussières de mica blanc. La peinture est passée en une seule rouche fine; elle est noire I tes opaque, et s'écaille facilement.

30 Fond annulaire de rase gris-monochrome. inv. C1/208 (1ig. 21. no:3:3) : forme indéterminable. la pàte, gris-rlair, fine, est tris vacuolaire; elle est assez tendre, laisse des traces au toucher et se raye a l'ongle. Le dégraissant présente trois éléments : des particules de calcaire, des inclusions rouges el une fine poussiere de mira blanc.

fo Fragment de fibule de bronze. inv. C1/267 (lig. 21, no :34) : il s'agit d'une moitie de ressort, a arbalite, long. Étant donnée la minceur du fil de bronze enroulé autour de l'axe, nous devons avoir affaire a un lype a faux ressort. 

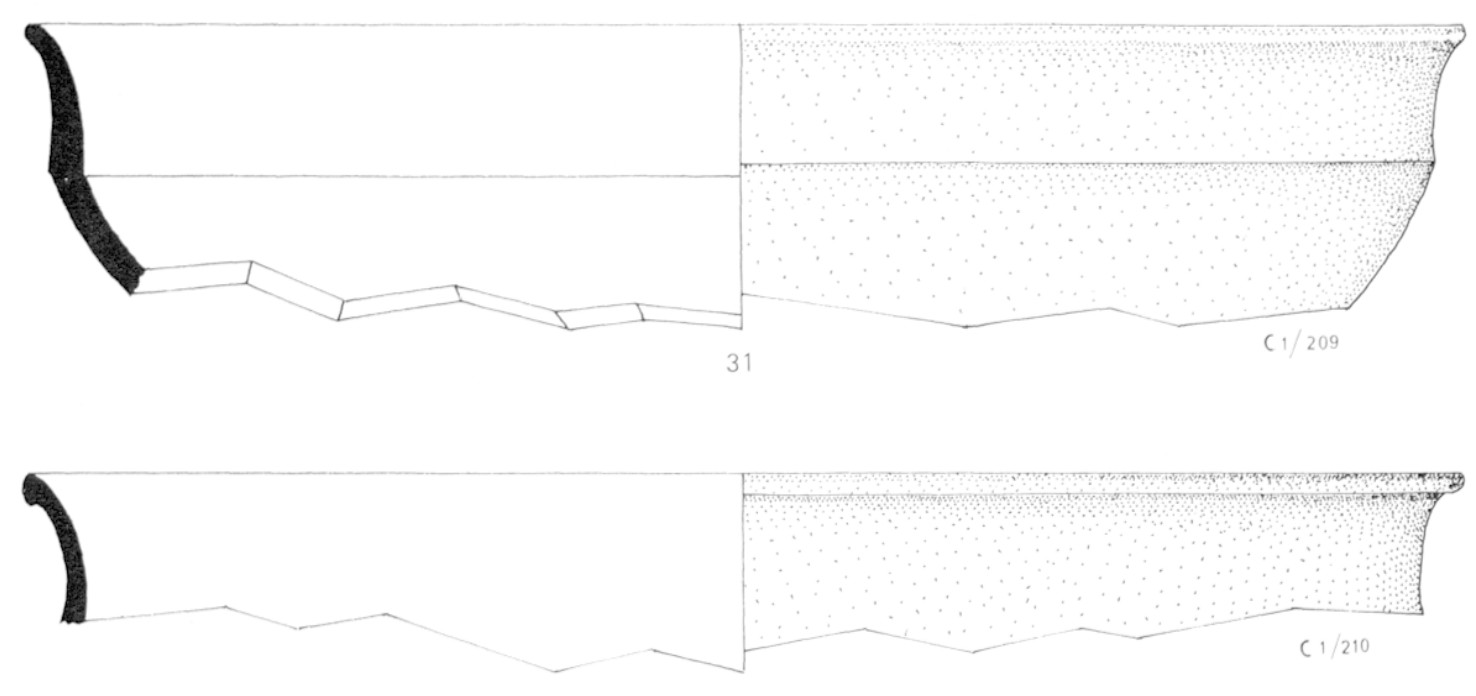

32

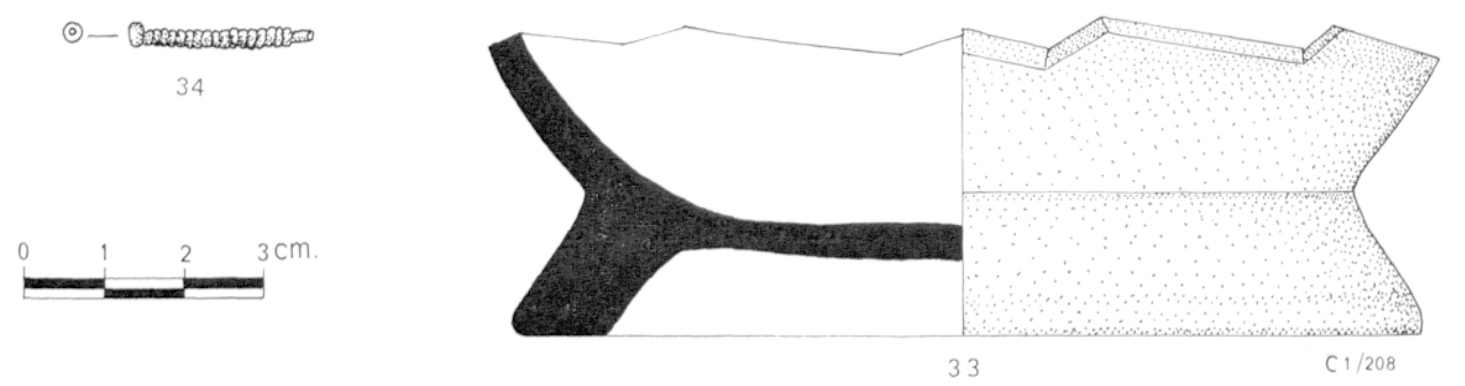

21 Trouvailles hors tombe : $1^{\circ} 31:$ bord de coupe attique ; $n^{\circ} 32$ : hord de coupe ionio-atlique ; $n^{\circ} 33$ : fond de vase gris monochrome; $n^{\circ} 3.4$ : fragment de fibule en bronze.

\section{IATATION RT GOMPARASONS}

Tombe 1. Le mobilier apporte des étéments de datation d'inégalde préeision selon les pièces. Ia coupe atlique (ins. (a)/4) trouve des correspondants dans un puits du vies.

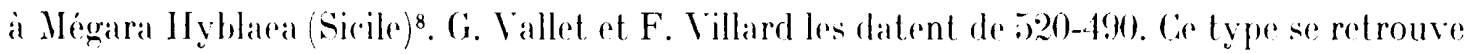
à la fin du vie s. a Thasos et, en de nombreux exemplaires, a Athènes durant la période $525-475)^{9}$. (on note également, dans les couches d'abandon du site voisin de La Liquière, la presenen de deux tessons à vernis noir présentant une teedhique identique, en ronnexion arec des roupes l'imitation B3:. Sur ee site on ne rencontre aurun matériel posterieur aux environs de 540. Par ailleurs l'oppidum de la Font du Coucou a livere des fragments a

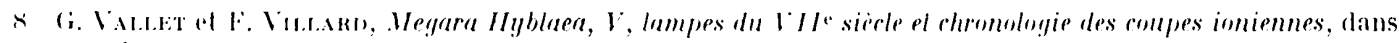

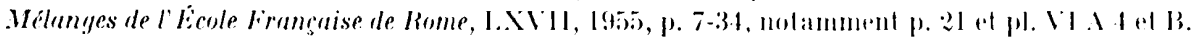

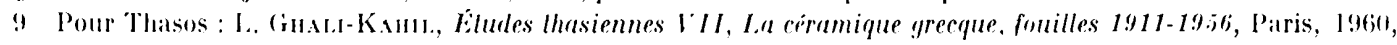

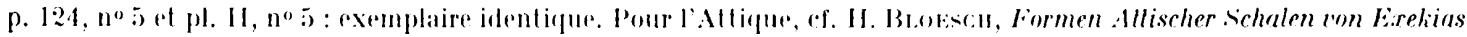

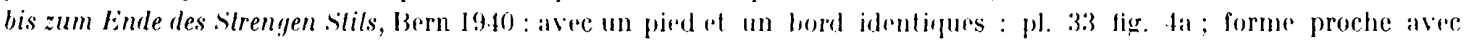
variante du pied : pl. 6 fig. 3 a et pl. 33 fig. 7. 
vernis noir de mème technique, et une imitation en céramique grecque d'occident de la mème forme. Cas témoins se trouvaient, dans une courhe datée de la deuxième moitié du vies.. en connexion ${ }^{10}$ aver une coupe de tradition B:211, des bords d'amphore ionienne à légraissant sablé, nt des bords d'amphore étrusque allongés ${ }^{12}$. La forme même de la roupe attique de la tombe 3 appartient à la série ca des roupes altiques à rernis noir datée de la fin du vies. el du début du ves.

Le vase en céramique non tournée (inv. (6)/1), bien que de forme simple, posside un similaire a Ampurias, dans l’incinération no 9 de la nécropole de la Muralla n.e. ${ }^{13}$ que II. Almagro situe vers ajol ou à une date légèrement postérieure.

Quant au vase en réramique grecque d'ocecident (inve (c) inédite et en tout ras originale. Pour une époque nettement antérieure, O. et .J. Taffanel ont reconnu cette forme en céramique non tournée lans la néropole du Cirand-Bassin I ${ }^{14}$. Le pied haut se retrouve dans cette même catégorie de réramiques sur d'autres formes dans la nécropole du Grand-Bassin ${ }^{15}$ et dans celle de Saint-Julien-de-Pézenas ${ }^{16}$, et, en céramique tournéc à pâte grise, dans les nécropoles de Saint-Julien-de-Pézenas et du Grand-Bassin II ${ }^{17}$. Le décor ondé, encadré de stries, est par ailleurs très courant dans la deuxième moilié dur vies.

Enfin, le couteau en fer de la tombe 1 est semblable an type dre couteau arque que loon rencontre all Grand-Bassin $\mathrm{II}^{18}$ et a Saint-Julien-dr-Pézenas ${ }^{19}$ depuis le début du ve s. Il se diftérencie neltement par contre du type droit du Cirand-Bassin I²0.

Tombe 3. Le fond d'amphore étrusque de la tombe 3 appartient au type le plus réeent de la série de forme pansue at pâte pulvérulente. Ce type semble apparaître all début du $v^{\mathrm{e}} \mathrm{s}^{21}$ et durer juscqu'au ve s. arece évolution de la lère. L'absence de celle-ci sur l'exemplaire de la Bergerie Hermet ne permet guère plus de précision.

10 Fouille (:1-1970, concher diabanton.

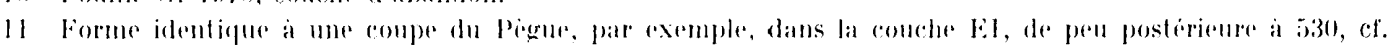

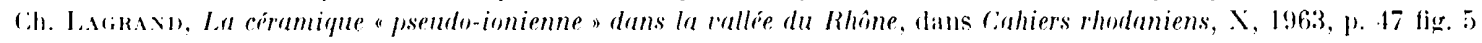
$11^{0} \stackrel{2}{2}$.

$1:$ Voir ci-dessons, mite? 21 .

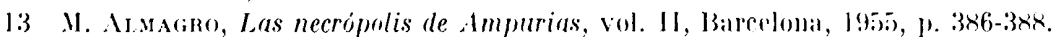

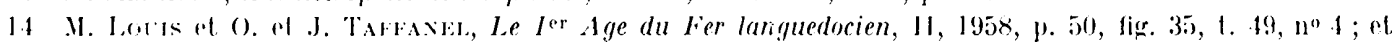

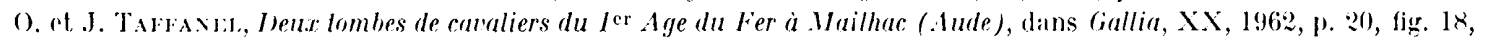
$n^{\circ} 38$ et p. 21, fig. 19, no 53 .

15) Combreux excmples dans les deux ouvrages ciles ci-dessus, note 14.

16. J. Giry, La nécropole próromaine de Saint-Julien (commune de Pézenas, Héraull), dans Revue d'iludes

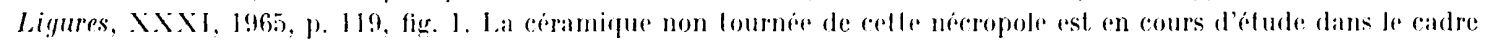
d'me lliese de ll Ie cyele B. I)edel'.

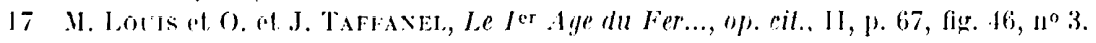

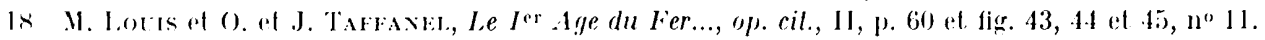

19 J. (iny, la necropole préromaine de saint-Julien..., loc. cil., p. 151, 1. 115 et 117 ; p. 202-203, 1.214.

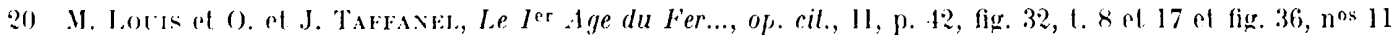

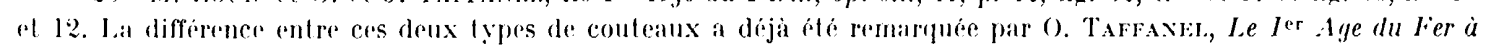
Mailhac (. lude), dans Cahiers Ligures de préhistoire el darchéologie, 11, 1962, p. 162.

21 Jes foublles de Vaunage ont permis d'etablir une lypologre probiminaire des amphores eltusques que nous pouvons résumer comme suit : un premier lỵpe qui apparaît au vio s. et disparaît peu apres 600, présente pour caracleristiques essentielles une live boudinee, un profil pansu et un fond plat. La pate en est dure et sablee, soit jaune verdale, soit rose. la paroi est mince. In deuxieme lype qui apparaît egalement au vac s. mais dure jusque vers jogo, a une levere boudinie, un profil egalement pansu, un fond en obus ; il présente une pate noire a l'interieur de la paroi, rouge vers la surface; a l'extirieur, la surface porte parfois un congobe creme ; la paroi est epaisse. In troisieme lype, 
L'urne non tournée de la fig. 9) no 7 (inv (:2/35) rst rourante en Languedoe entre le $\mathrm{v}^{\mathrm{e}}$ s. et le $\mathrm{II}^{\mathrm{e}}$ s. avant J.-C. ; rependant. sur cette forme, le fond reux est un indiere d'anciennetize La finition tres soignée de la surface (état de surface no 2), ainsi que l'égal aménagement du col et de la panse, va dans le mème sens ${ }^{23}$, tandlis que les exemplaires plus tardifs:24 se distinguent fortement par un aménagement different des surfaces : col lissé, panse prignére. linfin, le léeror que porte le vase de la Bergerie Hermet est le seul type de déeor incisé connu dans la région all vi $\mathrm{e}^{\mathrm{e}}$ s. Il disparait apres la fin da ve s. ${ }^{25}$.

Deux vases de la nécropole saint-Julien-de-Pezenas $\left(\mathrm{vi}^{\mathrm{e}} \mathrm{s}\right)^{26}$ sont exarctement identiques a l'ossuaire (inv. (92/32), avec des rapports hauteur sur diametre maximum tres semblables de 129 et 141 . On peut citer encore un vase lésererement antérieur dans la nécropole de Recobre à Quarante (Hérault)2

Le vase (ins. (2) [3:3) de la fig. 9, no 6 cat également tres courant. On le retroure en

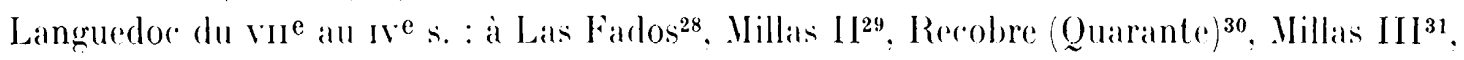
Ensérune (tombe 64)32, Vié-Cioutat ${ }^{33}$. In vase de forme at de rapport identiques, bien que de dimensions supérieures, existe dans la nécropole de Saint-Julien-de-Pézenas ${ }^{34}$. Rappelons que le vase non tourne de la tombe 1 de la Bergerie I Irrmet (inv. (5)/l) semble avoir la mème forme.

Le type de bracelet-armille plat, à section rectangulaire, sans être tres abondant,

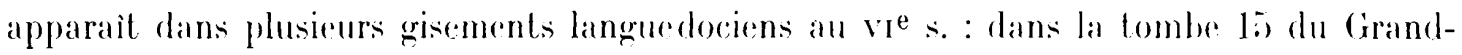

qui apparaît vers 600 el disparaîl vers 550 , presente une leve et une technique identique, mats un profil très efrile et un fond pointu. Enfin, un quatrieme tyee, de forme el daspect identipue an type 3 , se differencie par une lever allonge a profil en "amande"; ce lype apparait vers 550 et dure jusque vers la fin du ve s. les types 2at.3, durant le premier quart du vies., sont tous deux presents dans l'épave d'Antibes : cf. F. B1:xort, Recherches sur l'hellénisalion du Midi de la (iaule, Aix-en-Provence, 1965, p. 53 et pl. 41, fig. 3 et 4. Seul le lype 3 est present a lezenas dans les tombes de la premiere moitie du vie s. On rencontre sur ce mème gisement quelques bords du type 1, hors tombe. En Vaunage, sur loppidum de la lent du Coucou, occupe durant la deuxieme moitio du ve s., le seul lype represente est le lype 4 ; alors que, au contraire, a la liquiere ente 6.5 at 500, on ne rencontre que les lypes 1 at 3. Enfin, dans le grisement situe au pied de llauressip, de nombreux bords du tỵe 4 sont en comnexion avec des fragments de céramique altique a figures rouges dalés des dernieres anneres du ve s. av. J.-l:.

22 Voir par exemple le vase $1 \times 10$ de la lombe 197 de la necropole de Sainl-Julien-de-Pézenas illerault, datable du res. : exemplaire different cependant par le conlact col-panse qui est adouci.

23 Sur le vase cite precedemment, note 2:, on remarque un mème amenagement des surfaces. Voir encore

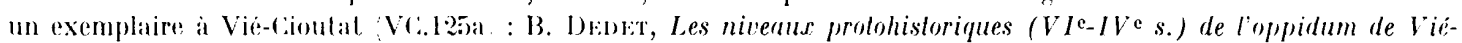
Cioulut, loc. cit.

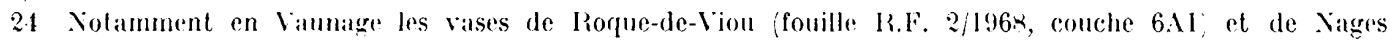
fouille AXIII2, phase Nages 1, 300-250).

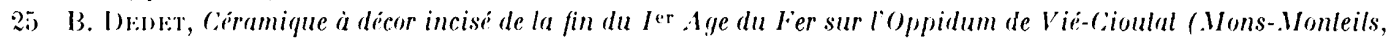
Gard), dans Cellicum XXIII, Congris de Tarbes 1969 fous presse.

26 Tombe 16 , vase $\mathbf{1 5 6}$ et lombe 109 , vase $A 29 \times$ bis tris semblables.

27 J. (inrs, Vécropole à incinération de Recobre à Quarante (Itéraull), dans Cahiers ligures de préhisloire el d'archéologie, 9, 1960, p. 147-197, en particulier fig. 6, nº 13.

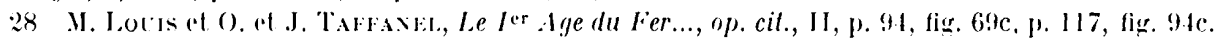

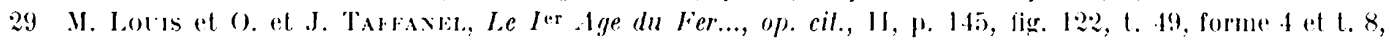
forme 10.

30 J. Gins, Vécropole à incinéralion de kecobre..., loc. cit., p. I×6, fig. 57, $1^{\circ} 2$.

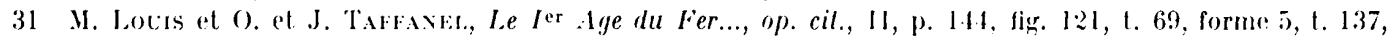
forme 9 , t. 124 , forme 11 et 19 .

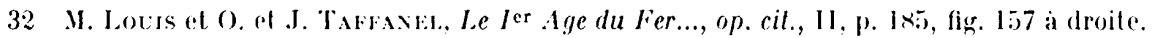

33 B. I) sons, Céramique dile indigrne du II Age du Fer sur l'Oppidum de Vie-Cioulat (.Mons-Monteils, (iard), dans Cahiers Ligures de préhistoire el darchéologie, 17, 1968, p. 187, fig. 11, 110 10 e p. 188, fig. 12.

34 Tombe 61, vase 1201 : hauteur : 0,21 m, rapport hauteur sur diametre maximum : 116. 
Bassin II ${ }^{35}$, dans la néropole de Saint-Julien-dr-Pézenas ${ }^{36}$, ainsi que dans le gisement

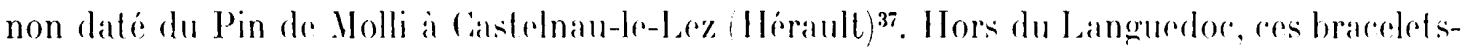
armilles sont presents en grand nomber of souvent en place dans les inhumations hallstattiennes de la nécropole des Jogasses a Chonilly (Marne ${ }^{38}$. In exemplaire tout à fait identique se trouve unfin rans la tombe 12:3 de la nécropole d'Agullana (Gerona, Expange $)^{39}$.

L'absence de la tête el du ressort de la fibule (inv. (2/8) ne permet pas des comparaisons completes. Par analogie on peut lui supposer deux types de ressort : un ressort bilatéral à nombre limité de spires, ou bien un ressort à arbalète long. Dans le premier cas, des exemplaires semblables proviennent de la nécropole de Saint-Julien-de-P'ézenas et datent du vie s., et sourent mème de la première moitié de ce siècleto. Dans le second cas, on rencontre ce type dans la Lombe 17 du Cirand-Bassin II"1 dans la Lombe de Corno-Lauzo à Pouzols- Minervois (Aude), bien datée des environs de 340 ${ }^{42}$ et dans plusieurs tombes de Saint-Julien-de-Pézenas ${ }^{43}$. S'il s'agit d'un exemplaire à faux ressort ornemental du type de celui provenant de la grotte du Travers a Montelus (Gard), et qui est aussi celui des spécimens de la tombe 17 du Cirand-Bassin II, il pourrait dater selon Ch. Lagrand de

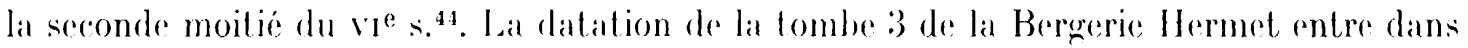
le $v^{e}$ s. et plus précisément dans la seconde moitie de ce siècle.

T'ombe 4. Elle est relativement bien daté grâce au bord d'amphore de type ionien dont la forme se retrouve datus la tombe de Corno-lauze to en connexion arec une coupe ionienne B:2 (580-540), une roupe atlique des petits-maitres (530-510), et, comme nous venons de le voir, une fibule a arbalite. On rencontre surtout ce lype d'amphore en grand nombere sur l'oppidum de la font du Coucou dans la deuxième moitié du vie s.

Les bracelets, identiques. pour la plupart, à ceux de la tombe 3, appellent les mèmes comparaisons, faites a ce propos. Cette sépulture se situerait done chronologiquement dans la deuxième moitié du $\mathrm{VI}^{\mathrm{e}} \mathrm{s}$.

T'ombe 2. Pour la dater, nous ne disposons que des comparaisons du vase non tourné avee des trouvailles faites sur l'oppidum de Nages qui sont tout a fait identiques ${ }^{46}$. Elles

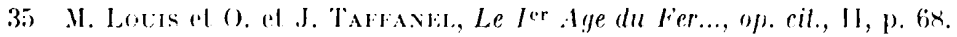

36 J. (ins, La necropole préromaine de siant-.Julien..., loc. cil., p. 160.

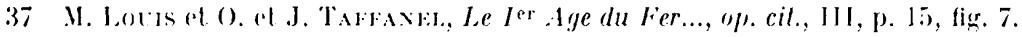

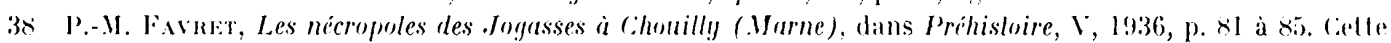
necropole ast datee de la lin du Hallshall.

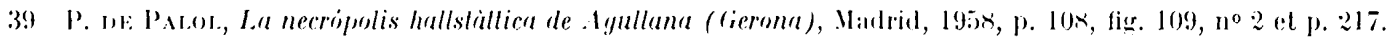
Cet exemplaire unique a Agullana est associé a deux urmes qui rallachent la tombe les a la seconde phase de la

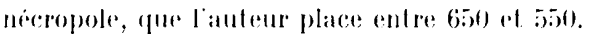

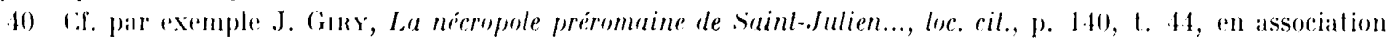
avec une amphore etrusque du lype 3 de la premiere moilie du ves.

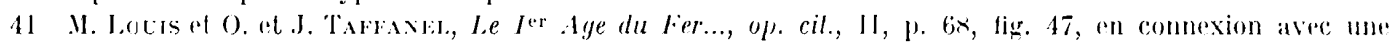
amphore massalible qui ne peut remonter an-dela de jö).

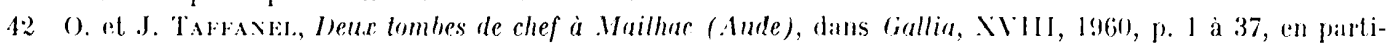
culier', fig. $1:, n^{\circ} 12$.

13. J. (iıy, La nécropole préromaine de saint-Julien..., loc. cil., p. 216, 1. 236.

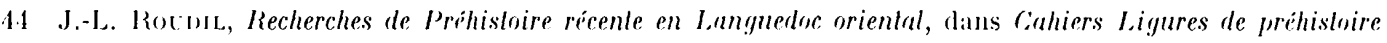
el d'archiologie, $11 \% 2,1965$, p. 172-173.

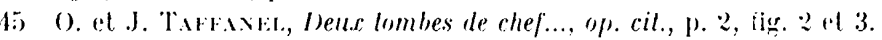

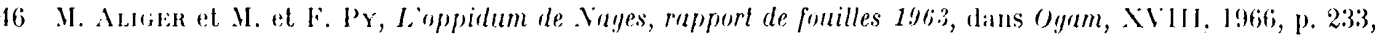
fig. $14, n^{\circ} 1$, p. 229 , fig. $12, n^{\circ} 26$ et p. 231 , fig. $13, n^{\circ} 43$. 
sont datées par connexion avere un nombreux numéraire at des céramiques a vernis noir

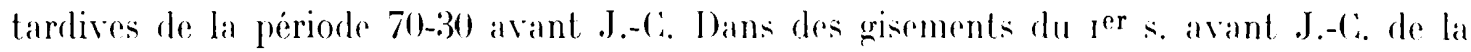
région, on trouve de nombreux similaires : a l'oppidum d Ambrussum (Villetelle, Hérault) ${ }^{47}$, dans la tombe de Saint-lalurent-des-Arbres (Gard) ${ }^{48}$, dans le puits no 7 de Cavaillon (Vaucluse), couche supérieure ${ }^{49}$. Le vase commun à pâte claire n’interdit pas de placer rette tombe aux alentours du milieu du I ${ }^{\text {er }}$ s. avant J.t.

Trourailles hors lomber. Les trouvailles hors tombe que nous arons signalées sont toutes contemporaines des tombes 1,3 at t. La plupart des categories quelles représentent se retrouvent, en effet, dans les tombes : coupe attique, céramique grise monochrome, et fibule a arbalète.

Le bord de coupe ionio-attique (inv. (1/210) se rattache aux productions marginales des coupes attiques à vernis noir ${ }^{-50}$. Si on ne peut lui assigner une origine précise, la datation en est certainement la mime.

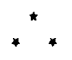

Le site de la Bergerie Hermet a d'abord été oceupe par un habitat de lype maillacien I. Sur l'emplacement même des habitations, à deux époques très nettement differentes, ont été établies des tombes à incinération.

A la première période appartiennent les tombes 1,3 et 4 et des témoins isolés, trouvés en surface apres défoncement du terrain. L'étude détaillée du mobilier des lombes 1 et 4 et des témoins de surface permet de les dater de la deuxième moitié du vi ${ }^{\mathrm{e}}$ s. La datation de la tombe 3 reste plus vague dans le $\mathrm{vI}^{\mathrm{e}}$ s. Cependant l'identité des bracelets-armilles des tombes 4 et 3 accrédite l'hypothèse d'une contemporanéité de ces trois tombes. Ciette hypothèse est fortement appuyé par le rapport qui existe entre ces tombes et l'oppidum tout proche de la Font du Coucou, ou a été reconnue une oceupation de la deuxième moitié du $\mathrm{VI}^{\mathrm{e}}$ s. Ces trois incinérations et l'oppidum semblent done former un ensemble. La tombe 2 par contre, datable du milieu du $\mathrm{I}^{\mathrm{er}}$ s. avant J.-C. est localement isolée.

Deux problèmes se posent a propos de la necropole de la Bergerie Hermet. Tout d'abord, localement, ses rapports avec les gisements protohistoriques de Vaunage; et, plus généralement, la place de la petite nécropole du vi s. dans l'ensemble du Premier Age du Fer languedocien. En V'aunage, l'ensemble formé par l'oppidum de la Font du Coucou et les tombes 1, 3 et 4 de la Bergerie Hermet permet de combler le hiatus qui existait entre la fin de l'occupation de l'oppidum de la liquière et la premiere occupation de l'oppidum de Mauressip, c'est-à-dire entre 5jo cet 500.

47 Renseignement communifur par le fouilleur, J.-L. Fiches, que nous remercions ici.

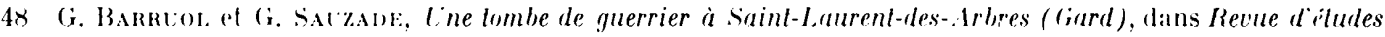
Ligures, XXXV, 1969 Hommaye à F. Benoîl, $1 \mathrm{IJ}, 1972$, p. 59-60.

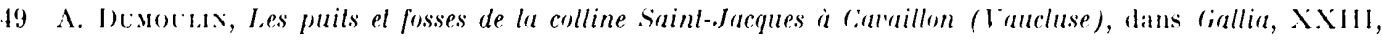
1965 , p. 21 , fig. $26: 1, \mathrm{~d}$ et $\mathrm{f}$.

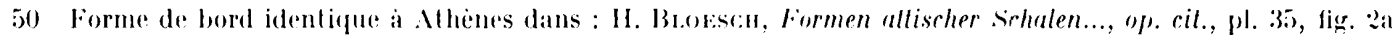
et 2 c et pl. 36, fig. ta 14 . 
La tombe du Ier s. avant .J.-C. est à rapprocher de plusieurs tombes isolées de mème époque trourées en différents lieux de la plaine de Vaunage : tombe de Boissierespe et tombe des Conclamines a Xages ${ }^{52}$. Les habitats contemporains en Vaunage sont : loppidum de Mauressip (dernière occupation) et l'oppidum de Xages phase III moyen et II f final).

En Languedoc oriental, entre Hérault et Rhòne, les tombes 1, 3 et 4 de la Bergerie Hermet sont les premières tombes plates a incinération de cette époque ${ }^{53}$, signalées. L'identité du matériel, et semble-t-il des rites, les rattache aux nécropoles a incinération du Languedoc occidental54. Plus précisément ces tombes sont contemporaines de certaines incinérations du Grand-Bassin $I^{55}$, de la tombe de Corno-Lallzo ${ }^{56}$ et d'un rertain nombre de tombes (? ? j7 détruites de Saint-Julien-de-Pézenas.

\section{Bernard Deset et Wichel PY.}

Anvexi:

\section{Eilude anlluropologitine}

Le mobilier anthropologique provenant des sépultures de la Bergerie Hermet, dont B. Dedet et. II. Py ont bien voulu nous confier l'élude, est évidemment réduit puisqu'il s'agit de tombes à incinération. Il se répartit comme suit.

Tombe 1 : le mobilier osseux est particulièrement restreint, et ne comporte que des fragments de diaphyse en fort mauvais état ; le seul fragment inléressant est un morceau de frontal, montrant

51 W. P'Y, La lombe de Boissières (Gard), dans Cahiers Ligures de préhistoire el d'archéologie 'sous presse: .

52 .I. Alritir, Nages (Gitrd), des origines à la fin de l'ire antique, dans Cellicum XVI, 1967, p. 53, pl. 24, fig. 46.

53 le tanguedoc oriental etait, en effet, totalement demuni de tombes plates à incincration pour toute la

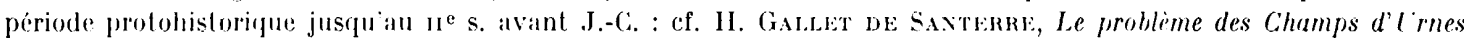
dans le Gard, dans Bulletin de la Société d' histoire el d'archéologie de Beaucaire, $\mathrm{n}^{\circ} 7,196: 3$, sans pagination.

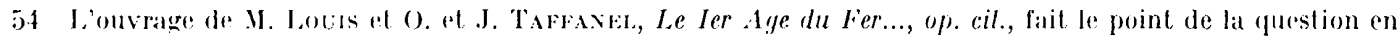
195x. Depuis lors ont éte déconvertes ou rendues publiques les necropoles suivantes dans l'ordre de leur publication':

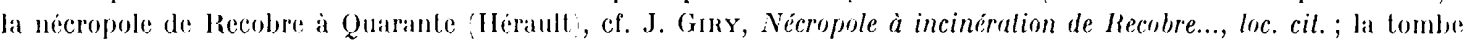

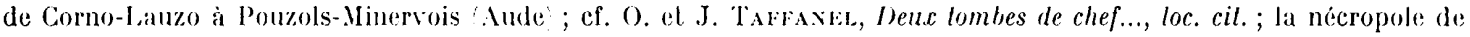
Vendres (Herault, cf. P. Aва zir, Vécropole du I er Age du Fer à Vendres (IIéraull), dans Bullelin de la Société préhislorique fransise, LVIH, L961, 3/1, p. 151-162; la necropole de Bonne-Terre a Tourbes Hérault, cf. J. Giry, Nécropole de Bonne-Terre à Tourbes (IÍrrull), dans Ciahiers Ligures de préhistoire et darchéologie, 10/1, 1961, p. 128-145; la nécropole de la Ballonette a Servian Héraull, cf. H. Prabss at J. Arxar, saluvelage diune nécropole de la civilisation

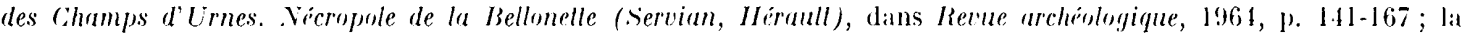
tombe de Mas-Saintes-Puelles Aude, cf. A. Soctoe ol J. Vian, Wobilier d une lombe à incinéralion de Mas-SaintesP’uelles (.1ude), dans Cahiers Ligures de préhistoire el d'archéologie, 13/1, 1964, p. 161-172. ; la nécropole de SaintJulien-de-P'ezenas (Herault, ef. J. Gır, Vécropole préromaine de saint-Julien..., loc. cit.; la nécropole d'olonzace

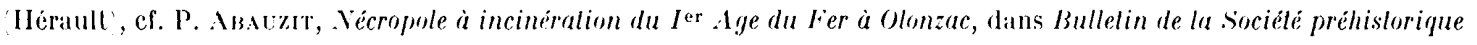
française, LXIV, 1967, p. 810-818.

55 En particulier, les tombes 14 et 17 de cette necropole, cf. M. Lovis el o. et J. Tarraxer., Le I er Age du Fer..., op. cil., p. 67 et 68 , et fig. 45,46 el 47.

56 O. et J. Tarianis, Deux tombes de chef..., loc. cit.

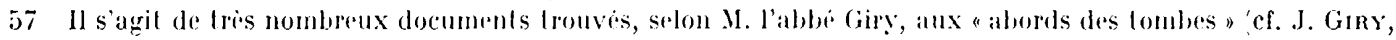
La nécropole de Saint-Julien..., loc. cit.) et notamment la ceramique atlique a figures noires coupes a yeux, etc.:. 
sur sa face interne la base du sinus sagillal médian du cràne, et sur sa face externe un métopisme tris net. L'épaisseur de l'os interdit de considerer qu'il s'agit d'un enfant de moins de six ans, et la présence de la suture métopique n'est done pas physiologique.

Tombe 3 : le mobilier osseux est plus important ; la crémation est très incomplète, mais les os sont trìs franmentés. Les fragments de cràne, nombreux et d'épaisseur variable, montrent tous des sutures non synostosées, ce qui implique qu'il s'agit d'un adulte jeune, vraisemblablement dans la troisième décennic, ou d'un adolescent. I.e splanchnocràne est représenté par un fragment d'arcade zygomatique, ainsi qu'un fragment de mandibule porlant les alvéoles des deux prémolaires inférieures G. Deux racines dentaires ont pu également ètre identifiées.

Les os longrs sont seulement représentés par des fragments de diaphyse, notamment de péroné et la partie proximale d'un radius ( $i$, portant la tète et la Lubérosité bicipitale. Nous avons pu identifier un scaphoïde du tarse, ainsi que quelques vertébres : les deux masses latérales de l'atlas, l'apophyse odontoïde de l'axis et le corps presque intact d'une vertèbre cervicale; de plus, un corps presque complet de vertỉbre dorsale, de hauteur peu élevée et montrant des plateaux mal soudés, pourrait confirmer l'hypothese d'un adulte jeune ou plutòt d'un adolescent.

Quelques éléments de faune, non brûlés, n'ont pu ètre identifiés.

Tombe $t$ : le mobilier est très irrégulièrement incinéré ; les fragments de cràne montrent tous des sutures disjointes et indiquent donc, iri aussi, un sujet jeune. Mris a part une racine dentaire et quelques fragments de diaphyse, le seul elément identifiable est un condyle mandibulaire.

L'élude de ce mobilier, restreinte en raison de la nature des sépultures. a montré que les tombes 3 el 4 contenaient les restes de deux adultes jeunes ou d'adolescents, et que le sujet de la tombe 1 était porteur d'un métopisme.

\section{Henri Duday.}

